University of Rhode Island

DigitalCommons@URI

Open Access Master's Theses

2015

\title{
ION-BOMBARDMENT OF NICKEL (110) AT ELEVATED TEMPERATURE
}

Vijay Kumar Peddinti

University of Rhode Island, vijay@ele.uri.edu

Follow this and additional works at: https://digitalcommons.uri.edu/theses

\section{Recommended Citation}

Peddinti, Vijay Kumar, "ION-BOMBARDMENT OF NICKEL (110) AT ELEVATED TEMPERATURE" (2015). Open Access Master's Theses. Paper 550.

https://digitalcommons.uri.edu/theses/550

This Thesis is brought to you for free and open access by DigitalCommons@URI. It has been accepted for inclusion in Open Access Master's Theses by an authorized administrator of DigitalCommons@URI. For more information, please contact digitalcommons-group@uri.edu. 
ION-BOMBARDMENT OF NICKEL (110) AT ELEVATED TEMPERATURE BY

VIJAY KUMAR PEDDINTI

A THESIS SUBMITTED IN PARTIAL FULFILLMENT OF THE REQUIREMENTS FOR THE DEGREE OF

MASTER OF SCIENCE

IN

PHYSICS

UNIVERSITY OF RHODE ISLAND

2015 
ProQuest Number: 1586565

\title{
INFORMATION TO ALL USERS
}

The quality and completeness of this reproduction is dependent on the quality and completeness of the copy made available to ProQuest.

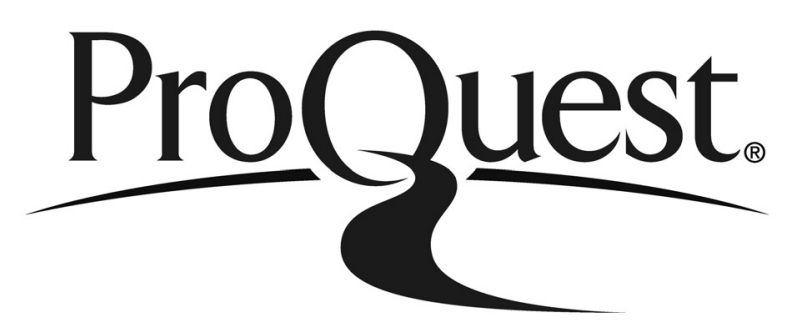

Distributed by ProQuest LLC (2015).

Copyright of the Dissertation is held by the Author unless otherwise noted.

This work may be used in accordance with the terms of the Creative Commons license or other rights statement, as indicated in the copyright statement or in the metadata associated with this work. Unless otherwise specified in the copyright statement or the metadata, all rights are reserved by the copyright holder.

This work is protected against unauthorized copying under Title 17, United States Code and other applicable copyright laws.

Microform Edition where available @ ProQuest LLC. No reproduction or digitization of the Microform Edition is authorized without permission of ProQuest LLC.

\author{
ProQuest LLC \\ 789 East Eisenhower Parkway \\ P.O. Box 1346 \\ Ann Arbor, MI 48106 - 1346 USA
}


MASTER OF SCIENCE THESIS

$\mathrm{OF}$

VIJAY KUMAR PEDDINTI

APPROVED:

Thesis Committee:

$\begin{array}{ll}\text { Major Professor } & \text { David R. Heskett } \\ & \text { Ramdas Kumaresan } \\ & \text { Leonard M. Kahn } \\ & \text { Nasser H. Zawia } \\ & \text { DEAN OF THE GRADUATE SCHOOL }\end{array}$

UNIVERSITY OF RHODE ISLAND

2015 


\begin{abstract}
The goal of this thesis is to study the behavior of ion-induced defects at the $\bar{Y}$ point on the Ni (110) surface at elevated temperatures. The electronic structure of the surface is examined using inverse photoemission spectroscopy (IPES), and the geometric structure is observed using low energy electron diffraction (LEED). These measurements lead to a better understanding of the surface properties.

The clean Ni (110) surface exhibits a peak $\sim 2.6$ eV above the Fermi level, indicating an unoccupied surface state near the $\bar{Y}$ point of the surface Brillouin zone (SBZ). Defects are induced by low energy ion bombardment at various temperatures, which result in a decrease of the peak intensity. The surface state eventually disappears when bombarded for longer times. We also observed that the surface heals faster when the crystal is being simultaneously sputtered and annealed at higher versus lower temperature. Finally the data for annealing while sputtering versus annealing after sputtering does not seem to exhibit much difference.
\end{abstract}




\section{ACKNOWLEDGMENTS}

My research would not have been possible without support from several individuals. First, I would like to thank and express my deepest gratitude to my major professor, Dr. David Heskett. He is definitely one of the best mentors a student can ask. From my experience with experimental work during this thesis, I have developed even more respect and admiration for all the experimental researchers; as obtaining good repeatable results is not guaranteed, despite substantial amount of meticulous prep work. Dr. Heskett's patient mentorship made it possible to get through such challenging days. Without his support, this thesis would not have been possible. I am fortunate for the opportunity to work with him. Thanks to Ben for his time and efforts for showing me the ins and outs of the lab equipment. Thanks to Mauricio for reviewing my thesis as well. Additionally, I would like thank Anton for his assistance in the lab.

I would like to extend my deepest gratitude to all the professors in my thesis committee that includes Dr. Godi Fischer, Dr. Leonard Kahn, and Dr. Ramdas Kumaresan. I would like to thank them for their time, insightful comments and for their support throughout the thesis.

I have thoroughly enjoyed my time in both the Physics and Electrical engineering departments, and I would like to thank the graduate students with whom I have interacted through the years. I would like to thank the Electrical engineering department for providing teaching assistantship over the years and also the Air Force Office of Scientific Research (AFOSR) funding (from Dr. Kumaresan, my major professor in the Electrical engineering department), which helped me in completing my masters and Ph.D. in Physics and Electrical engineering, respec-

tively. Thanks to Linda Connell, our department secretary, student helpers, Steve Pellegrino, and Dave Notarianni for their assistance. 
I am grateful to my parents, and brother for their support. Their love and support is very much appreciated. I also would like to acknowledge Larry McLaughlin, Scott Lachance and their families for their love and support. This paragraph is dedicated to all those people who I could not mention due to space constraints and to all the experimental researchers. 


\section{DEDICATION}

This thesis is dedicated to family and friends 


\section{TABLE OF CONTENTS}

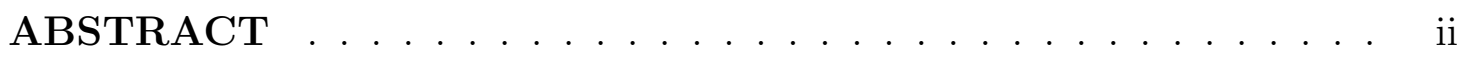

ACKNOWLEDGMENTS ................... iii

TABLE OF CONTENTS $\ldots \ldots \ldots \ldots \ldots \ldots \ldots$

LIST OF FIGURES . . . . . . . . . . . . . . vii

\section{CHAPTER}

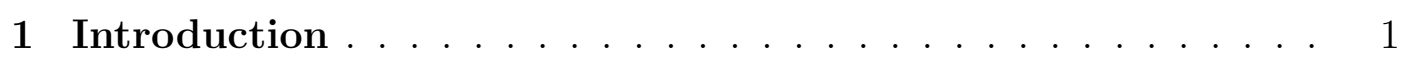

1.1 Sputtering/ion bombardment . . . . . . . . . . . 4 4

1.2 Annealing . . . . . . . . . . . . . . . . 7

List of References . . . . . . . . . . . . . . . . . . . 7

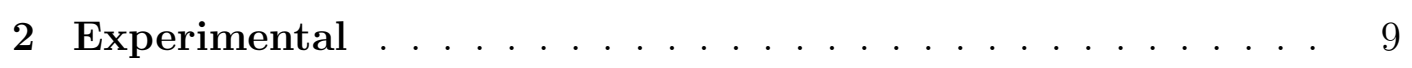

2.1 Inverse photoemission spectroscopy (IPES) $\ldots \ldots \ldots \ldots$

2.2 Low energy electron diffraction (LEED) . . . . . . . . . . 14

2.3 Experimental Setup . . . . . . . . . . . . . . . 16

List of References . . . . . . . . . . . . . . . . . 2 20

3 Results and Discussion . . . . . . . . . . . . . 21

3.1 Results and Discussion . . . . . . . . . . . . . . 21

3.2 Inverse photoemission spectroscopy (IPES) Results . . . . . . 22

3.3 LEED results . . . . . . . . . . . . . . . . . . . 31

3.4 Summary and Future work . . . . . . . . . . . . . . 34

List of References . . . . . . . . . . . . . . . . . 34 


\section{Page}

\section{APPENDIX}

Additional reference material . . . . . . . . . . . . 35

A.1 Ni sputtering result at 170 and $300 \mathrm{~K}[1] \ldots \ldots$

A.2 Face centered cubic (FCC) lattice $\ldots \ldots \ldots \ldots$

A.3 Other IPES results . . . . . . . . . . . . . . . . . . . . 39

List of References . . . . . . . . . . . . . . . . . 4 40

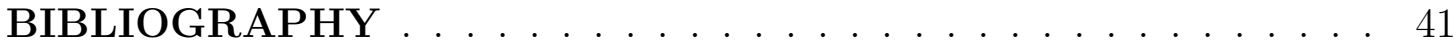




\section{LIST OF FIGURES}

Figure

Page

$1 \quad$ Energy versus momentum (k) for Ni (110) showing the unoccupied region above $E_{f}$ [6]. $S_{1}, S_{2}$ are related to surface states and $B_{1}, B_{2}$ corresponds to Bulk. Energy versus momentum band diagram for Nickel is shown in the appendix Figure A.5. .

2 (a) Nickel band structure between L and X [10]. The E(k) parabola is for a free electron in a constant potential. (b) Nickel (110) surface brillouin zone (SBZ) projection to the bulk brillouin zone [11]. The notation follows the standard terminology, where $\Gamma, \mathrm{W}, \mathrm{L}, \mathrm{U}$, corresponds to the bulk structure, and $\bar{\Gamma}, \bar{Y}$, $\bar{X}, \bar{S}$ (with an overhead bar) corresponds to the surface. For an FCC (110) crystal $\Gamma$ - origin, L - $\left(\frac{1}{2}, \frac{1}{2}, \frac{1}{2}\right), \mathrm{X}-(100), \mathrm{W}-$ $\left(\frac{1}{2}, 1,0\right)[12] \ldots \ldots \ldots \ldots \ldots \ldots$. . . . . . . . . . . . .

3 Bulk Brillouin zone projections for face centered cubic (FCC) structure [7]. . . . . . . . . . . . . . . . . 5

4 (a) illustrates a typical contaminated surface. (b) sputtering (to remove contamination) is illustrated. . . . . . . . . . . 5 5

$5 \quad$ Sputtering removes the contamination particles, however it generates a rough surface. . . . . . . . . . . . . 6

6 Different surface defects (a) Schottky (b) interstitial (c) Frenkel defects $[12] \ldots \ldots \ldots \ldots$

7 A smooth Ni surface is obtained by annealing. . . . . . . . . . . 7

$8 \quad$ IPES spectrum schematic of the unoccupied density of states [2]. 9

$9 \quad$ Energy level diagram of photoemission (PES) and inverse photoemission $(\mathrm{IPES})[3] . \ldots \ldots \ldots$

10 A different illustration of photoemission (PES) versus inverse photoemission (IPES) [4]. A result showing the complementary nature of PES and IPES for Co is shown in the appendix (figure A.6). . . . . . . . . . . . . . . 11

11 A typical IPES seup with a Geiger-Müller Tube [2]. . . . . . . . 12 
12 Comparison of the detectable photon energy range for the $\mathrm{CaF}_{2}$ and $\mathrm{SrF}_{2}$ windows [8]. . . . . . . . . . . . . 12

13 Schematic of a low energy electron diffraction (LEED) system $[11] \ldots \ldots \ldots \ldots \ldots \ldots$

14 A commercial low energy electron diffraction (LEED) apparatus manufactured by Omicron (image reference: [12]). . . . . . . 15

15 A LEED pattern for Cu FCC (110) lattice [7], for Ni would be $0.89 \AA^{-1}$ and $1.2607 \AA^{-1}$. . . . . . . . . . . . . . 16

16 Lab ultra-high vacuum (UHV) chamber. . . . . . . . . . 17

17 Sample mounted in the chamber using a sample holder, which is made with a tantalum foil folded around tungsten wires. . . . 18

18 The plot presents an inverse photoemission spectra after sputtering (with and without annealing). The x-axis is energy in $\mathrm{eV}$, with 0 being the the Fermi edge $E_{f}$ and normalized counts (normalized to the average current developed across the sample) on the y-axis. The annealed crystal shows a clean peak around $2.6 \mathrm{eV}$, whereas the unannealed spectrum does not exhibit any such peak. The other peak $\sim 0.3 \mathrm{eV}$ is primarily caused by transitions to the vacant d-band [1]. Also, the solid lines are the smoothed counterparts of the data shown by markers alone. . . 23

19 Inverse spectra for intermediate sputtering. The plots correspond to: clean, 5 minute sputter at $1 \times 10^{-6}$ Torr, 5 minute sputter at $5 \times 10^{-6}$ Torr, and 30 minute sputter. . . . . . . . 24

20 Inverse spectra for sputtering at $2.5-2.5-2.5-2.5-5$ minute intervals at $2 \times 10^{-6}$. The plots correspond to: clean, 2.5 minute sputter, 2.5 and 5 minute sputter. . . . . . . . . . . 24

21 The plots shows the ratio of the surface area with respect to the clean surface on the y-axis; the area of the clean surface is normalized to 1 . On the $\mathrm{x}$-axis is the cumulative sputtering time. The plot shows the decrease in the surface peak intensity as a function of cumulative sputter time. . . . . . . . . .

22 Inverse spectra when the sample is annealed at $35{ }^{\circ} \mathrm{C}(308 \mathrm{~K})$ and $59{ }^{\circ} \mathrm{C}(332 \mathrm{~K})$ while sputtering for 10 minutes at $5 \times 10^{-6}$ Torr. . . . . . . . . . . . . . . 27 
23 Inverse spectra when the sample is annealed at $47^{\circ} \mathrm{C}(320 \mathrm{~K})$, $71{ }^{\circ} \mathrm{C}(344 \mathrm{~K}), 132{ }^{\circ} \mathrm{C}(405 \mathrm{~K})$ for 10 minutes at $5 \times 10^{-6}$ Torr.

24 The plot presents temperature on the $\mathrm{x}$-axis, and the ratio of the surface peak intensity with respect to $132{ }^{\circ} \mathrm{C}(405 \mathrm{~K})$ for a 10 minute sputter at $5 \times 10^{-6}$ Torr. . . . . . . . . . . . . 28

25 Inverse spectra when the sample is annealed at $35^{\circ} \mathrm{C}(308 \mathrm{~K})$ during a 10 minute sputter at $5 \times 10^{-6}$ Torr, compared to a 10 minute sputter at $5 \times 10^{-6}$ Torr followed by a 10 minute anneal

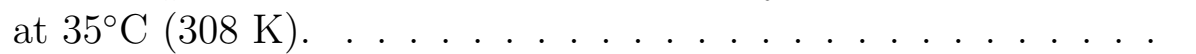

26 Inverse spectra when the sample is annealed at $23^{\circ} \mathrm{C}(296 \mathrm{~K})$, during a 10 minute sputter at $5 \times 10^{-6}$ Torr, compared to a 10 minute sputter at $5 \times 10^{-6}$ Torr followed by a 10 minute anneal at $23^{\circ} \mathrm{C}(296 \mathrm{~K}) . \ldots \ldots \ldots \ldots$

27 Inverse spectra when the sample is annealed at $29{ }^{\circ} \mathrm{C}(302 \mathrm{~K})$, during a 10 minute sputter at $5 \times 10^{-6}$ Torr, compared to a 10 minute sputter at $5 \times 10^{-6}$ Torr followed by a 10 minute anneal at $29{ }^{\circ} \mathrm{C}(302 \mathrm{~K}) \ldots \ldots \ldots \ldots$

28 LEED image without annealing after a 30 minute sputter at 5 $\times 10^{-5}$ Torr. . . . . . . . . . . . . . . . . . 31

29 LEED image after a 5 minute annealing. . . . . . . . . . . . . . 32

30 LEED image after a 10 minute sputter at $5 \times 10^{-6}$ Torr. . . . . 32

31 LEED image after a 10 minute sputter at $5 \times 10^{-6}$ Torr while annealing at at $35^{\circ} \mathrm{C}(308 \mathrm{~K}) \ldots \ldots . \ldots . \ldots 33$

32 LEED image after a 10 minute sputter at $5 \times 10^{-6}$ Torr followed by a 10 min anneal at $35^{\circ} \mathrm{C}(308 \mathrm{~K})$. . . . . . . . . . . . . . 33

A.1 B. Young's Ni (110) results for 170 and $300 \mathrm{~K}[1]$. . . . . . . . 35

A.2 FCC lattice showing the (100), (110), (111) faces and unit cells

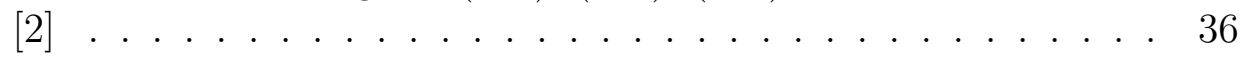

A.3 Ni (110) surface brillouin zone (SBZ) projection to the bulk brillouin zone $[3] \ldots \ldots$. . . . . . . . . . . . 37

A.4 FCC lattice showing the 110 face and unit cell [3] . . . . . . 37 
A.5 Energy versus momentum band diagram for Nickel [4] . . . . . . 38

A.6 Band mapping of photoemission (PES) versus inverse photoemission (IPES) for $\mathrm{Co}(0001)$ sample (Cobalt). For the same conditions, in IPES shows the unoccupied states corresponding to the minority $3 \mathrm{~d}$ bands, whereas the PES provides information about the majority spin states $[5] \ldots \ldots$. . . . . . . 38

A.7 Inverse spectra when the sample is annealed at $23{ }^{\circ} \mathrm{C}(396 \mathrm{~K})$ and $40{ }^{\circ} \mathrm{C}(413 \mathrm{~K})$ for 10 minutes at $5 \times 10^{-6}$ Torr. . . . . . . . 39

A.8 Inverse spectra when the sample is annealed at $71{ }^{\circ} \mathrm{C}(444 \mathrm{~K})$ and $52{ }^{\circ} \mathrm{C}(425 \mathrm{~K})$ for 10 minutes at $5 \times 10^{-6}$ Torr. . . . . . . 39

A.9 inverse spectra for different detection angles . . . . . . . . . . 40 


\section{CHAPTER 1}

\section{Introduction}

The goal of this thesis is to develop a better understanding of ion-induced surface defects at a higher temperature. Nickel is selected for the analysis as it exhibits clear distinct surface peaks in the unoccupied region as detected by inverse photoemission spectroscopy (IPES). Clean Ni (110) shows an unoccupied electronic surface state with energy $\sim 2.6 \mathrm{eV}$ above the Fermi level, near the $\bar{Y}$ point of the surface brillouin zone (SBZ). In order to create surface defects the Ni sample is ion-bombarded at different elevated temperatures, followed by the electronic and geometric structure study. The thesis is organized as follows:

- Chapter 1 provides a brief overview on the Nickel (110) surface and the techniques used for the surface analysis.

- Chapter 2 provides a description of inverse photoemission spectroscopy (IPES) and low energy electron diffraction (LEED), two characterization tools used in the thesis. This chapter also discusses the experimental chamber setup.

- Chapter 3 presents results and discussion, followed by a brief discussion of future work.

The surface structure is typically the starting point which leads to a wide range of understanding of surface phenomena [1]. Changes at the surface influence structural, vibrational, and magnetic properties among others. Surface electronic states and defects have been of considerable interest for decades. It is important

to understand surface defects, as they are present while dealing with most real world surfaces, needed in developing advanced technologies. For example, surface 
defects are important to understand on semiconductors and electronics where the feature sizes are reducing dramatically. In some cases, we are dealing with 10-20 atoms (electrons) or less, and removing even two or three atoms could have a big impact.

This research work is an extension of prior work in Dr. Heskett's lab [2, 3, 4]. Prior work in the lab also includes surface analysis on Copper, Nickel and adsorption on metals. In the $\mathrm{Cu}(110)$ study, it was found that copper didn't show any decrease in surface state peak intensity at room temperature, however the peak decreased in intensity at $170 \mathrm{~K}$ [4]. This was attributed to self-annealing of Copper at room temperature. From a more recent study on Ni (110), B. Young found that at room temperature $(300 \mathrm{~K})$ Ni needed 10 times the sputtering dosage to generate similar results as at $170 \mathrm{~K}$ [5]. It was concluded that the $\mathrm{Ni}$ was exhibiting some (but not complete) self-annealing at room temperature $(300 \mathrm{~K})$ when compared to a lower temperature $(170 \mathrm{~K})$. Some of these results are shown in the appendix (Figure A.1). Along these lines, it would be interesting to see the temperature dependence of Nickel as well. Nickel was used for the study as it was readily available and also the $\mathrm{Ni}$ and $\mathrm{Cu}$ study is a good step before working with complex materials. Both $\mathrm{Ni}$ and $\mathrm{Cu}$ have a face centered cubic structure (FCC, shown in Figure A.4 in the appendix), and are d-band metals. The Nickel melting point is $1455^{\circ} \mathrm{C}(1728 \mathrm{~K})$; Copper's melting point is $1085{ }^{\circ} \mathrm{C}(1358 \mathrm{~K})$.

A clean $\mathrm{Ni}(110)$ surface exhibits a peak $\sim 2.6 \mathrm{eV}( \pm 0.2 \mathrm{ev}[7])$ above the Fermi level, indicating an unoccupied state near the $\bar{Y}$ point of the surface Brillouin zone (SBZ). Figure 1 highlights this surface state, $S_{1}$ along $\bar{Y}$. In the plot $S_{1}$ and $S_{2}$ are surface states, while $B_{1}$ and $B_{2}$ represent bulk state transitions.

This surface state is in the projection of the $L_{2^{\prime}}-L_{1}$ gap as highlighted in Figure 2, where (a) presents the Nickel (110) band between L and X (L - $\mathrm{C}-\mathrm{X}$ ) 


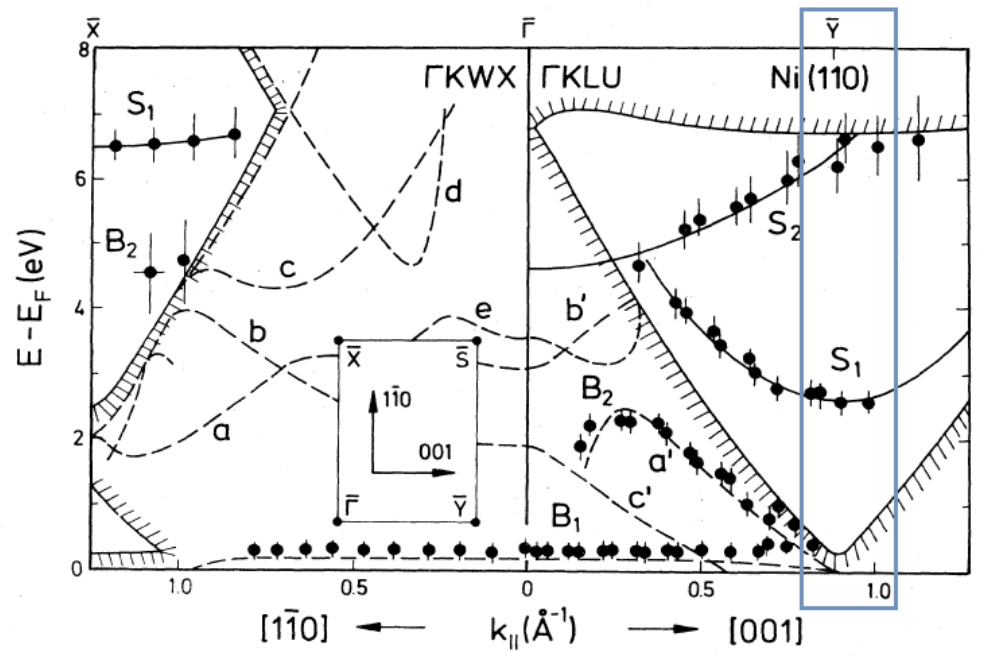

Figure 1. Energy versus momentum (k) for Ni (110) showing the unoccupied region above $E_{f}[6] . S_{1}, S_{2}$ are related to surface states and $B_{1}, B_{2}$ corresponds to Bulk. Energy versus momentum band diagram for Nickel is shown in the appendix Figure A.5.

and the $\mathrm{L}_{2^{\prime}}-L_{1}$ bandgap is highlighted, while (b) illustrates the bulk to surface projection. Even though theoretical analysis shows a continuous band in a metal by introducing a surface in a solid, the three dimensional symmetry is broken, giving rise to geometrical and electronic structure modifications [7]. These modifications result in different boundary conditions giving rise to otherwise forbidden electron states. Figure 2 (a) highlights two such forbidden states between $\mathrm{L}_{2^{\prime}}$ to $\mathrm{L}_{1}$ and $\mathrm{X}_{4^{\prime}}$ to $\mathrm{X}_{1}$. Unoccupied surface states are seen near the middle of these gaps [8]. These states are called Shockley surface states or crystal-induced states (where the gap is p-like at the bottom and s-like at the top; a detailed analysis is provided in $[9,8]$ using the multi-reflection approach and near free electron analysis). The $\mathrm{L}_{2^{\prime}}-\mathrm{L}_{1}$ gap extends from $\sim 0.9 \mathrm{eV}$ to about $+6.5 \mathrm{eV}$. The surface state is around $2.8 \mathrm{eV}$. The projected gap at $\bar{Y}$ is derived from the $\mathrm{L}_{2^{\prime}}-L_{1}$ gap, and the $\bar{X}$ corresponds to $\mathrm{X}_{4^{\prime}}-X_{1}$ gap. It is interesting to note that the $\mathrm{L}$ bandgap can be also sampled from $\bar{X}$ on the 100 plane and $\bar{\Gamma}$ on the 111 plane, as shown in Figure $3[7]$. 


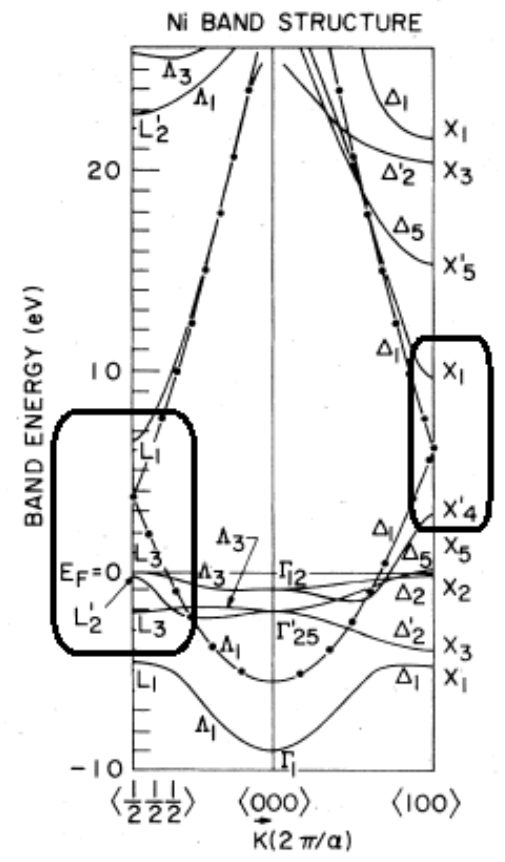

(a)

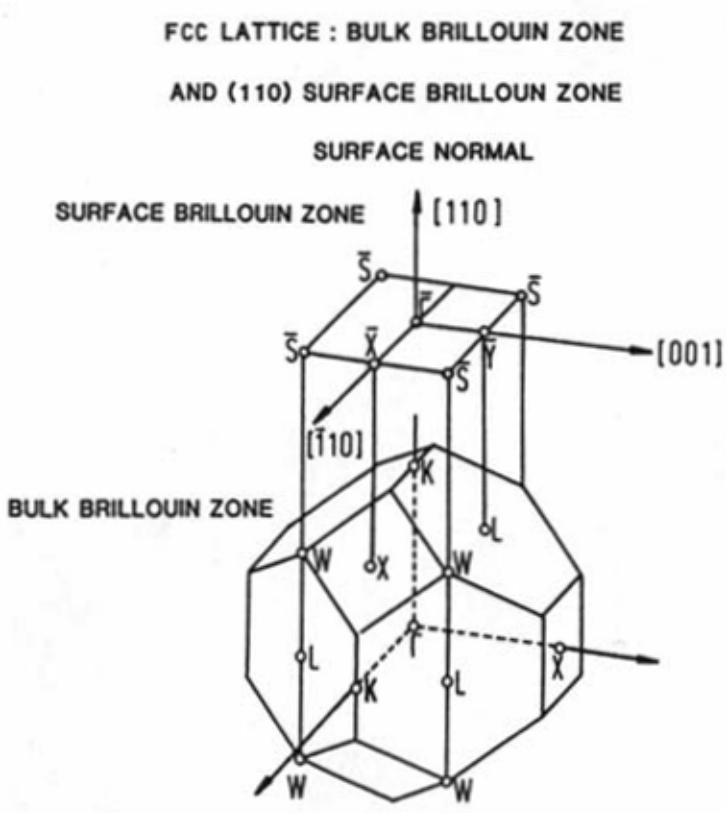

(b)

Figure 2. (a) Nickel band structure between L and $\mathrm{X}[10]$. The $\mathrm{E}(\mathrm{k})$ parabola is for a free electron in a constant potential. (b) Nickel (110) surface brillouin zone (SBZ) projection to the bulk brillouin zone [11]. The notation follows the standard terminology, where $\Gamma, \mathrm{W}, \mathrm{L}, \mathrm{U}$, corresponds to the bulk structure, and $\bar{\Gamma}, \bar{Y}, \bar{X}$, $\bar{S}$ (with an overhead bar) corresponds to the surface. For an FCC (110) crystal $\Gamma$ - origin, L - $\left(\frac{1}{2}, \frac{1}{2}, \frac{1}{2}\right), \mathrm{X}$ - (100), W - $\left(\frac{1}{2}, 1,0\right)[12]$.

The different techniques utilized in this thesis to study the Ni crystal are the following:

- Surface cleaning is performed by sputtering/ion bombardment and annealing.

- Surface characterization is by inverse photoemission spectroscopy (IPES) and low energy electron diffraction (LEED).

\subsection{Sputtering/ion bombardment}

In any surface characterization study, it is desirable to start with a clean crystal surface. Sputtering or ion bombardment is an important technique used for sample cleaning. It involves bombarding the sample with inert gas ions. Typically, 


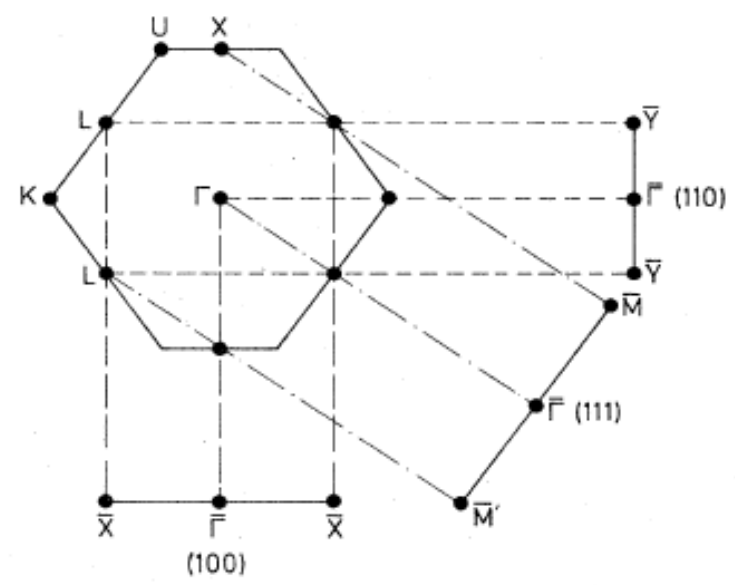

Figure 3. Bulk Brillouin zone projections for face centered cubic (FCC) structure [7].

Argon or Neon is used as they do not bind with the surface during the process. High energy particles are used to remove the unwanted impurities from the surface, while low energy particles are used to induce defects in a controlled environment. In our system, Argon (Ar+ ions) is used for this procedure.

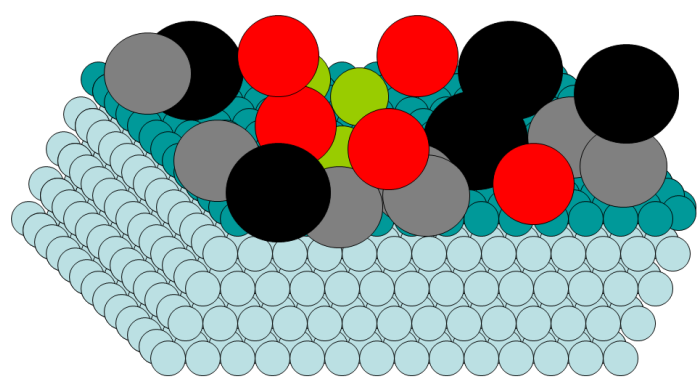

(a)

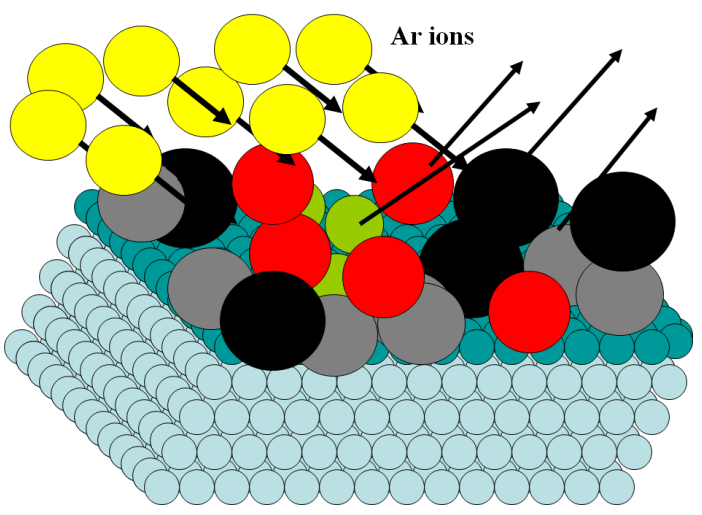

(b)

Figure 4. (a) illustrates a typical contaminated surface. (b) sputtering (to remove contamination) is illustrated.

Figure 4(a) illustrates a typical surface with contamination, particles due to oxidation, moisture, carbon dioxide and so forth collect over time. A clean crystal surface is obtained by sputtering. During this process even though the contamination particles are removed, a few layers of the crystal are also eliminated and 
result in a uneven surface, as illustrated in Figure 5.

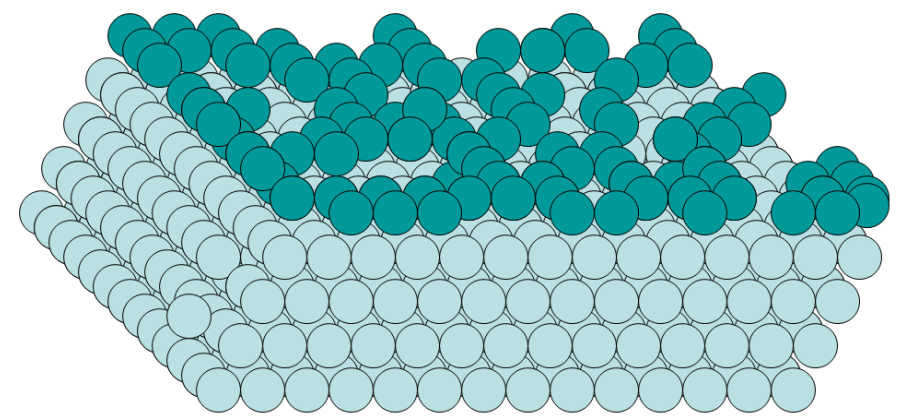

Figure 5. Sputtering removes the contamination particles, however it generates a rough surface.

The three simple defects produced by sputtering are vacancies or Schottky defects, interstitials and Frenkel defects, as shown in Figure 6. The first is missing atoms or vacancies, where atoms acquire energy from the incident energy particles and leave an interior lattice site to reach the crystal surface. These vacancies are also called Schottky defects. The second type is called interstitials: they are created when the atoms come to rest interstitially and perturb the lattice. The third one is referred as Frenkel defects, where the incident energy atoms generate vacancies and interstitials in the process. Hence these are also called Frenkel pairs $[13,12]$. Among these defects, surface vacancies are the most common defects generated by sputtering at low ion energies and small doses [14].

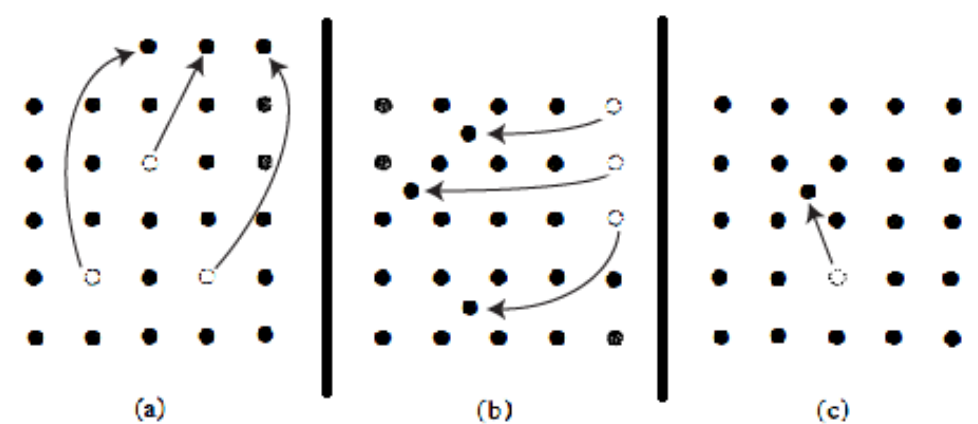

Figure 6. Different surface defects (a) Schottky (b) interstitial (c) Frenkel defects $[12]$. 


\section{$1.2 \quad$ Annealing}

After sputter cleaning, the sample is heated to a high temperature, approximately half the melting point, to remove the surface defects/vacancies. As illustrated in Figure 5, sputtering results in vacancies (surface defects) and an uneven surface. By annealing, most of these vacancies (surface defects) are healed by diffusion, thus resulting in a smooth, clean and healed surface. Figure 7 illustrates the surface after annealing.

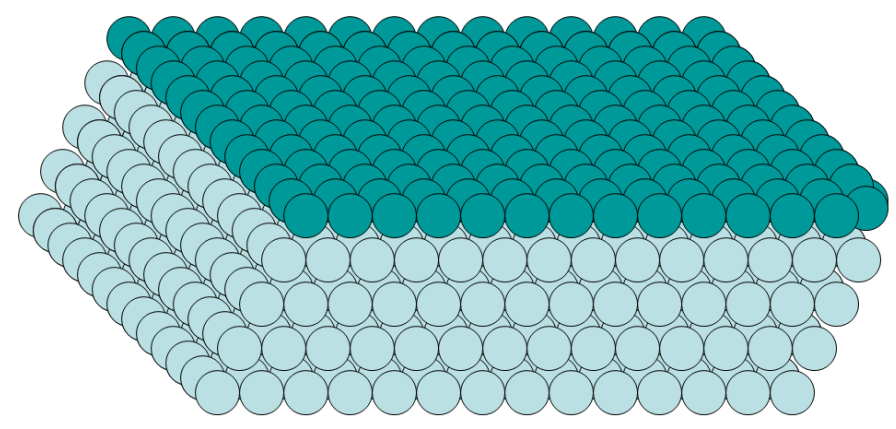

Figure 7. A smooth Ni surface is obtained by annealing.

Now we can study the properties of the sample. In this thesis, the two techniques used to study the crystal structure are inverse photoemission spectroscopy (IPES) and low energy electron diffraction (LEED). These techniques are discussed in the next chapter.

\section{List of References}

[1] D. P. Woodruff, "Solved and unsolved problems in surface structure determination," Surface Science, pp. 147-171, 2002.

[2] D. Tang and D. Heskett, "Unoccupied electronic structure of Na/Ni(111)," Physical Review B, vol. 47, no. 16, pp. 695-699, 1993.

[3] C. Su, D. Tang, and D. Heskett, "Two-dimensional unoccupied electronic band structure of clean cu (110) and $(1 \times 2)$ na/cu (110)," Surface science, vol. 310, no. 1, pp. 45-51, 1994.

[4] D. Heskett, D. DePietro, G. Sabatino, M. Tammaro, "Ion bombardmentinduced changes in the electronic structure of $\mathrm{Cu}(110)$ investigated with in- 
verse photoemission and computer simulations," Surface Science, vol. 513, pp. 405-411, 2002.

[5] Benjamin Young, "Electronic and Chemical Properties of Surface AND Interfacial Zones," Ph.D. dissertation, University of Rhode Island, Aug 2014.

[6] A. Goldmann, M. Donath, W. Altmann, and V. Dose, "Momentum-resolved inverse photoemission study of nickel surfaces," Physical Review B, vol. 32, no. 2, pp. 837-850, July 1985.

[7] A. Goldmann, V. Dose and G. Borstel, "Empty electronic states at the (100), (110), and (111) surfaces of nickel, copper, and silver," Physical Review B, vol. 32, no. 4, pp. 971-1980, August 1985.

[8] N. V. Smith and D. P. Woodruff, "Inverse photoemission from metal surfaces," Progress in surface science, vol. 21, no. 4, pp. 295-370, 1986.

[9] N. V. Smith, "Phase analysis of image states and surface states associated with nearly-free-electron band gaps," Phys. Rev. B, vol. 32, pp. 3549-3555, Sep 1985. [Online]. Available: http://link.aps.org/doi/10.1103/PhysRevB.32.3549

[10] F. J. Himpsel, J. A. Knapp, and D. E. Eastman, "Experimental energy-band dispersions and exchange splitting for Ni," Physical Review B, vol. 19, no. 6, pp. 2919-2927, 1979.

[11] Stephan Hüfner, Photoelectron Spectroscopy Principles and Applications. Springer, 2003.

[12] Gerald Burns, Solid State Physics. Orlando, Florida: Academic Press, Inc., 1985.

[13] J. J. Bellina and H. E. Farnsworth, "Ion Bombardment Induced Surface Damage in Tungsten and Molybdenum Single Crystals," The Journal of Vacuum Science and Technology, vol. 9, no. 2, pp. 616-619, 1972.

[14] M. A. Vasylyev, A. G. Blaschuk, N. S. Mashovets and N. Yu. Vilkova, "LEED study of $\mathrm{Ni}(100)$ and (111) surface damage caused by $\mathrm{Ar}+$ ion bombardment with low energy and small doses," Vacuum, vol. 57, pp. 71-80, 2000. 


\section{CHAPTER 2}

\section{Experimental}

This chapter presents an overview of the two surface characterization techniques used to study the $\mathrm{Ni}$ (110) surface: inverse photoemission spectroscopy (IPES) and low energy electron diffraction (LEED). In addition the experimental setup is also discussed in this chapter.

\subsection{Inverse photoemission spectroscopy (IPES)}

IPES provides information about the unoccupied or empty states between the Fermi level and vacuum levels in solids. In IPES, the sample is bombarded with a beam of electrons of known energy, $E_{k i n}$. Some of these incident electrons make a transition to a lower empty state above the Fermi level and a photon is emitted in

the process. A spectrum is obtained by sweeping the electron beam energy $E_{k i n}$ and monitoring the emerging photons of fixed energy, $\hbar \omega[1]$. The resulting plot mirrors the unoccupied density of states, as illustrated in figure 8.

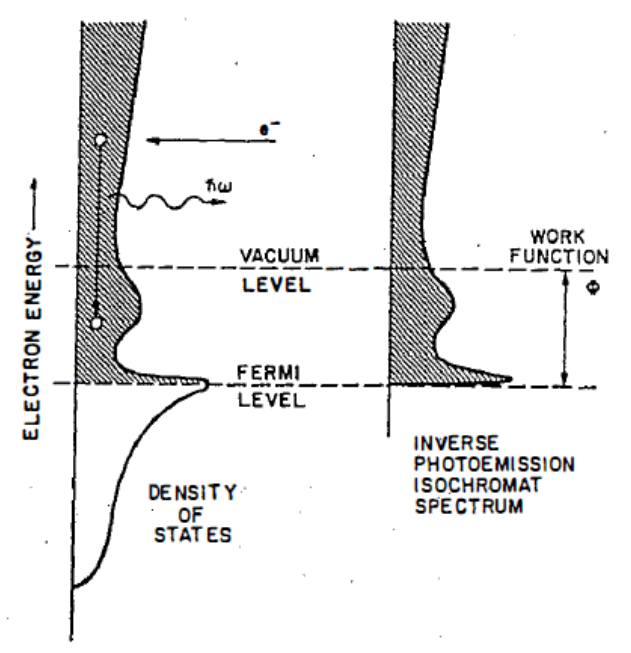

Figure 8. IPES spectrum schematic of the unoccupied density of states [2]. 


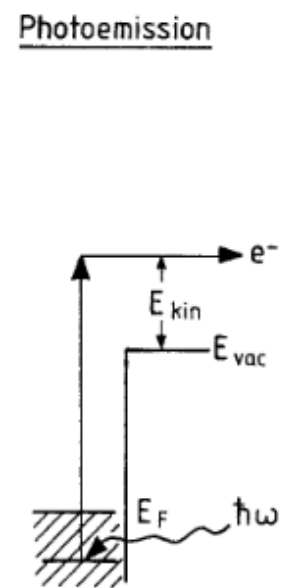

a
Inverse Photoemission

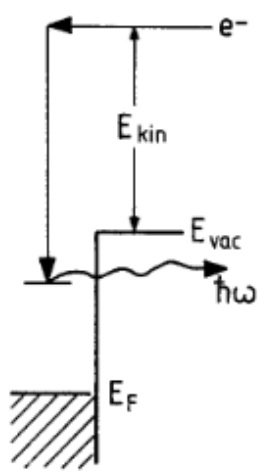

b

Figure 9. Energy level diagram of photoemission (PES) and inverse photoemission (IPES) [3].

Figures 9 and 10 show a comparison between photoemission and inverse photoemission. Photoemission provides information about the occupied states, whereas IPES measures the unoccupied states. Hence, these two techniques complement each other.

IPES is also known as bremsstrahlung isochromat spectroscopy (BIS), and in-fact, it is still referred as BIS when working in the x-ray region [2]. IPES gained popularity when BIS was reformed to use low energy $(\leq 1 \mathrm{keV})$ electrons and produce UV light, instead of using high energy electrons (on the order of $10 \mathrm{keV}$ ) and record X-rays. This modification enabled IPES to study surfaces [4]; in this sense BIS laid the foundation for IPES.

The key requirements of IPES are an electron source (also referred as an electron gun) and a photon detector. IPES can be operated in two modes: 1. Isochromat mode: where the incident electron beam energy, $E_{k i n}$, is varied, and the emitted photons at a constant energy, $\hbar \omega$, are detected. 2. Spectrograph mode: in this mode the incident electron energy, $E_{k i n}$, is fixed and the emitted photons are collected over a range of energies [1]. In this thesis, IPES is operated in the 
PHOTOEMISSION

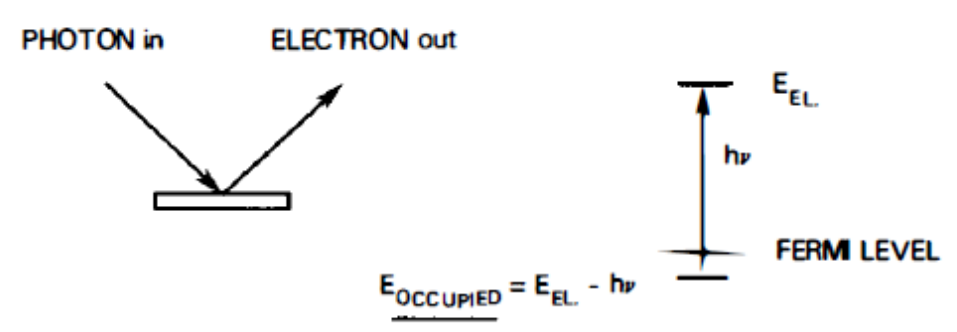

INVERSE PHOTOEMISSION (BREMSSTRAHLUNG SPECTROSCOPY)

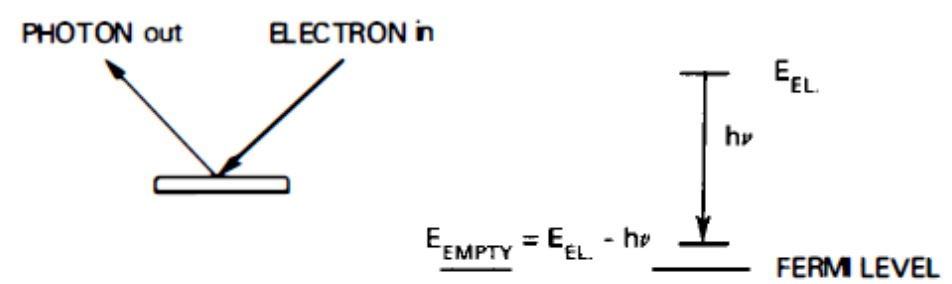

Figure 10. A different illustration of photoemission (PES) versus inverse photoemission (IPES) [4]. A result showing the complementary nature of PES and IPES for Co is shown in the appendix (figure A.6).

isochromat mode.

In our setup, we use a home built electron gun based on an Erdman-Zipf design [5] and a Geiger-Müller (GM) Tube, which is a popular choice as an isochromat photon detector. The GM counter is one of the oldest and a low cost radiation detector introduced by Geiger and Müller in 1928 [6]. Hans Geiger originally developed the principle; later Walther Müller collaborated to extend the detection to several types of radiation, such as alpha particles, X-rays, gamma rays to name a few. Figure 11 shows a typical GM construction and setup. It is a stainlesssteel tube typically (25 mm $\sim 1$ " in diameter) filled with Iodine and Helium. One 
end is sealed with a calcium fluoride $\left(\mathrm{CaF}_{2}\right)$ or strontium fluoride $\left(\mathrm{SrF}_{2}\right)$ window. The $\mathrm{CaF}_{2}$ (or $\mathrm{SrF}_{2}$ ) window provides the high energy cutoff, while the Iodine gas gives the low energy cutoff [7]. The combination of $\mathrm{CaF}_{2}$ window with Iodine provides detectable photon energy of $9.7 \mathrm{eV}$ and a bandwidth of $0.8 \mathrm{eV}$. Using a $\mathrm{SrF}_{2}$ window reduces the detectable photon energy to $9.5 \mathrm{eV}$ with a bandwidth of $0.4 \mathrm{eV}$, as illustrated in figure 12, which is a plot of the detectable photon energy range for $\mathrm{CaF}_{2} / \mathrm{SrF}_{2}$ windows.

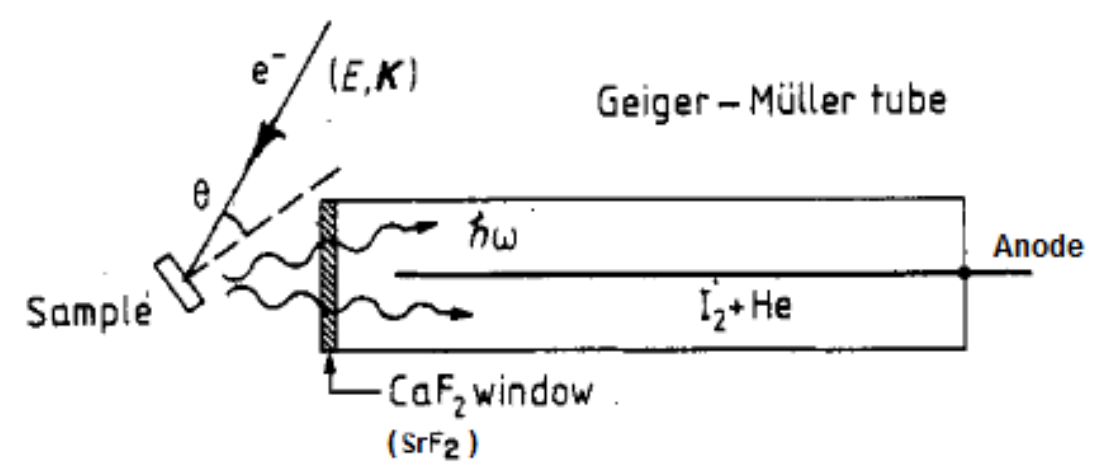

Figure 11. A typical IPES seup with a Geiger-Müller Tube [2].

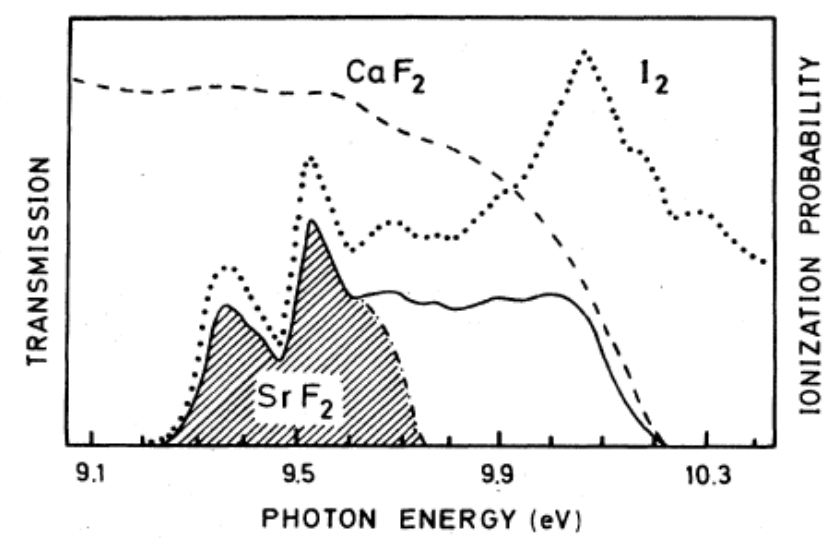

Figure 12. Comparison of the detectable photon energy range for the $\mathrm{CaF}_{2}$ and $\mathrm{SrF}_{2}$ windows [8].

The wall of the GM tube acts as a cathode and the wire in the center of the tube acts an anode. The GM tube uses Townsend avalanche to detect the 
radiation. The avalanche starts with a single electron, which gives rise to several excited gas molecules within a few nanoseconds. These excited molecules emit a photon when returning to the ground state, which generates free electrons by re-absorption or by hitting the cathode. The free electrons accelerated by the potential difference between the cathode and anode ionize the Iodine by collisions and the whole process repeats. The multiplication stops when a huge positive ion space charge is formed in the chamber, resulting in a reduced potential difference which cannot accelerate the free electrons. Another method to moderate these multiplications is to use a quencher, such as Helium [6]. The pressure of Helium should be moderated so it doesn't generate too many nor too low collisions.

Electrons of energy $E_{k i n}$ from an electron gun are incident on the sample at an angle $\theta$. The final energy state in terms of initial state energy is given by assuming bulk direct transitions $[2,9]$, and using the principle of conversation. We can write $E_{f}$ as the following

$$
\begin{aligned}
E_{f} & =E_{i}-\hbar \omega \\
& =E_{k i n}+\phi-\hbar \omega \\
E_{k i n} & =E_{f}+\hbar \omega-\phi
\end{aligned}
$$

Where $E_{f}$ is the final energy state,

$\phi$ is the work function (the minimum energy required to remove an electron from the Fermi energy of a solid), $E_{k i n}$ is the Kinetic energy of the incident electron, and $\hbar \omega$ is the detected photon energy.

The parallel component of momentum is conserved and the only relevant component. It can be computed using $E_{k i n}=\frac{\hbar^{2} \overline{\mathbf{k}}^{2}}{2 m}$, from which the momentum parallel 
to the surface can be obtained.

$$
k_{\|}=\sqrt{\frac{2 m}{\hbar^{2}}\left(E_{k i n}\right)} \sin (\theta)=\sqrt{\frac{2 m}{\hbar^{2}}\left(E_{f}+\hbar \omega-\phi\right)} \sin (\theta)
$$

Where $\theta$ is the angle of the electron incidence (or, in other terms, it is the angle between electron beam and sample normal).

\subsection{Low energy electron diffraction (LEED)}

Low energy electron diffraction (LEED) is one of the most common techniques used to probe the geometric structure of a crystal surface. It involves using a collimated beam of low energy electrons whose wavelength is close to the inter-atomic spacing of the crystal. When these electrons hit the surface, some of them backscatter and form a Fraunhofer diffraction pattern, which is the Fourier transform of the surface atom arrangement $[10,11]$. A clean surface displays a good periodic pattern.

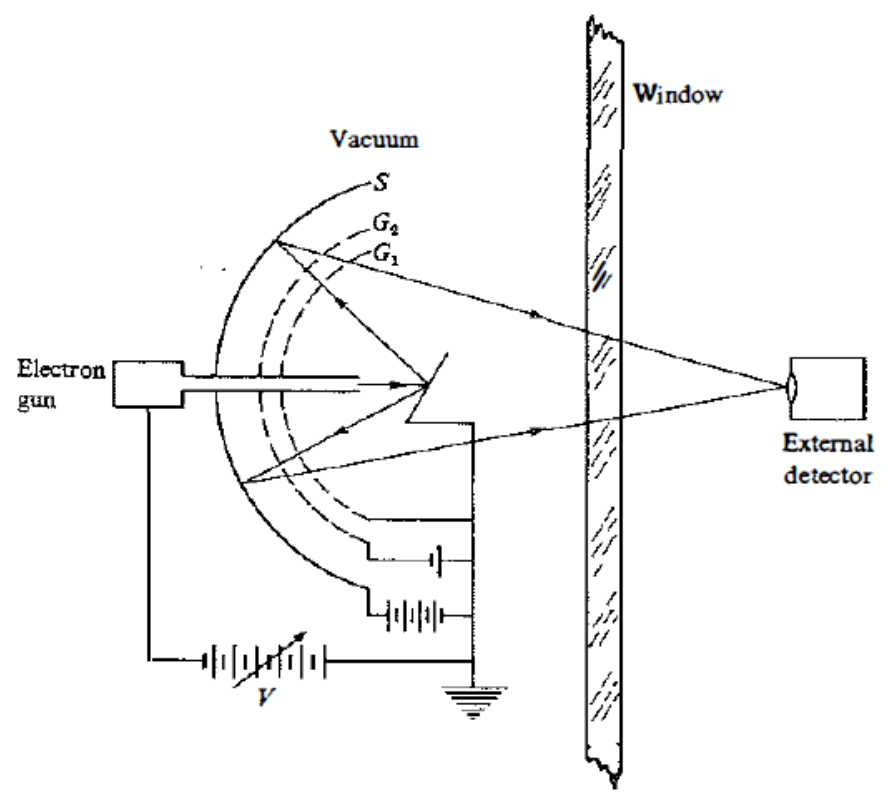

Figure 13. Schematic of a low energy electron diffraction (LEED) system [11].

A typical LEED setup is shown in figure 13. Low energy electrons are emitted from the electron gun, and a small fraction of them back-scatter and reach grid 
$G_{1}$. Only the elastically back-scattered electrons reach the second grid $G_{2}$, which finally excites the fluorescent screen $\mathrm{S}$. A detector, shown on the right, records this diffraction pattern. A popular LEED system manufactured by Omicron is shown in figure 14. The grids $G_{1}$ and $G_{2}$ can be seen on the top of the setup, where the fluorescent screen $\mathrm{S}$ is present, and the electron gun is located in the center.

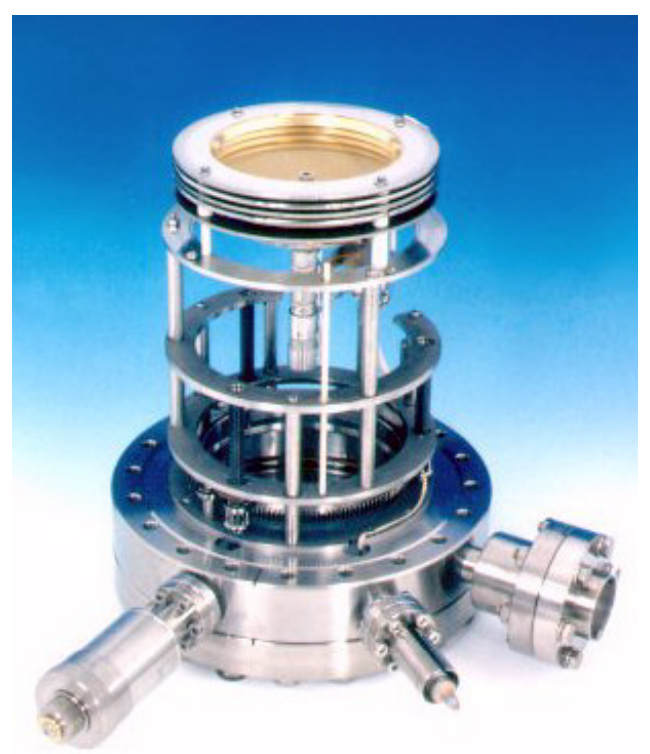

Figure 14. A commercial low energy electron diffraction (LEED) apparatus manufactured by Omicron (image reference: [12]).

In our setup, low energy electron diffraction (LEED) is performed using a 120 eV beam energy. In LEED, low energy electrons are used to obtain an image of the surface reciprocal lattice (figure 15) to reveal the geometric structure. These images provide accurate information on the atomic positions, symmetry, and degree of order of the surface.

For the surface pattern electrons, approximately $20-500 \mathrm{eV}$ is used. To compute the energy corresponding to the wavelength, let us start with the de Broglie relation $\lambda=\frac{h}{p}=\frac{h}{m v}$, where $h$ is the Plank's constant, $m=$ mass of the electron, 
$p=$ momentum. Using kinetic energy $E_{K}=\frac{1}{2} m v^{2}$, we can write $m v=\sqrt{2 m E_{K}}$. By substituting all the values in the de Broglie relation, $\lambda$ in terms of Angstrom $\AA$, can be expressed as $\lambda(\AA)=\sqrt{\left[\frac{150}{E(e V)}\right]}$. At an energy of $20-500 \mathrm{eV}, \lambda$ is on the same scale as the atomic distance.

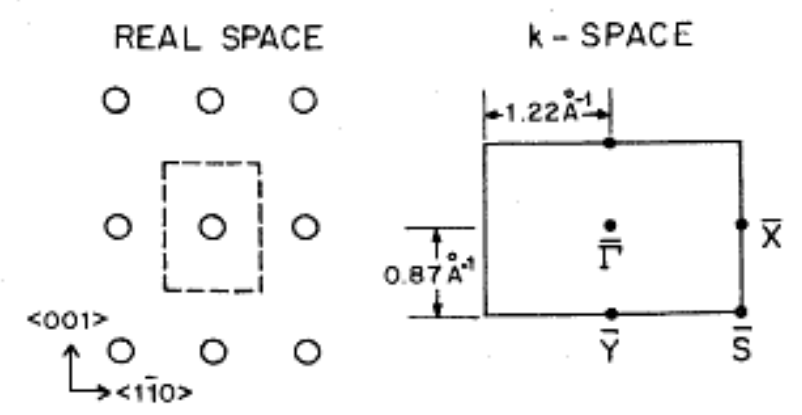

Figure 15. A LEED pattern for Cu FCC (110) lattice [7], for Ni would be 0.89 $\AA^{-1}$ and $1.2607 \AA^{-1}$.

Figure 15 shows the relationship between the real and reciprocal lattice or k-space for copper. The k-space dimensions for $\mathrm{Ni}$ would be $0.89 \AA^{-1}$ and 1.26 $\AA^{-1}$.

\subsection{Experimental Setup}

Figure 16 shows the experimental setup. All the measurements are conducted at a base pressure of $7 \times 10^{-11}$ Torr in the ultra-high vacuum (UHV) chamber. As a comparison, atmospheric pressure is 760 Torr. The chamber has two sections: in the top section sputtering and LEED are performed, while IPES is performed in the bottom part of the chamber. The chamber is equipped with a home-built angleresolved inverse photoemission spectrometer, Physical Electronics' LEED and ion source apparatus. In addition, the chamber is equipped with the standard pumps: turbo-molecular, ion, diffusion and roughing pumps. The turbo-molecular pump 
is ideal for pumping out gases such as Argon used for sputtering, while the ion pump primarily maintains the ultra high vacuum pressure. The pressure is sensed using an ionization gauge.

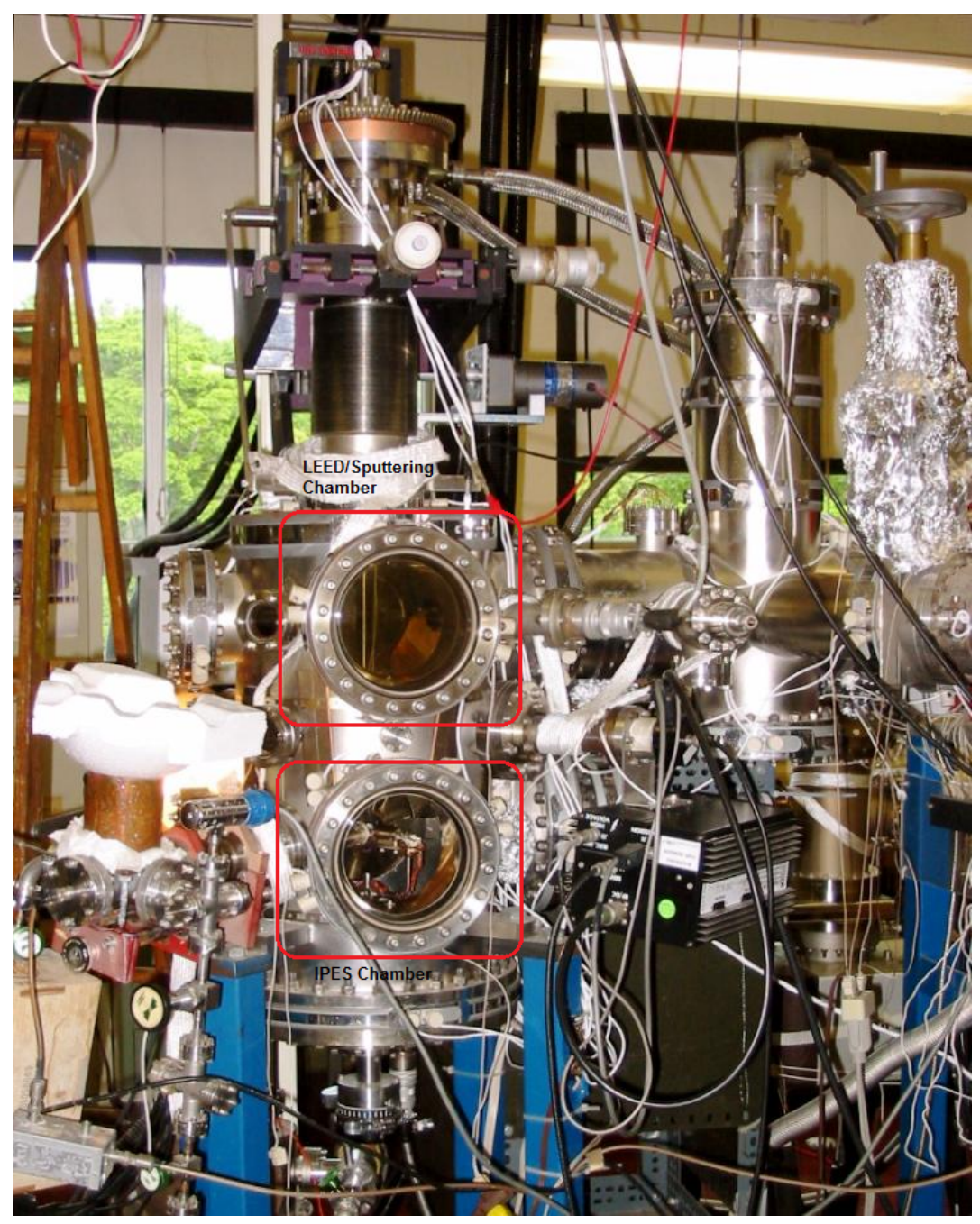

Figure 16. Lab ultra-high vacuum (UHV) chamber.

The sample is suspended in the chamber using a sample holder, as shown in figure 17, which is shown in the IPES position. On the right is the electron gun, and the left side is the photon detector. The photon detector is positioned to be within $2 \mathrm{~cm}$ of the sample surface at $45^{\circ}$ relative to the sample normal. The sample holder is a tantalum foil folded around tungsten wires, which are attached to the 
copper posts as shown in the figure. A high current is passed through the tungsten wires to heat the sample. The temperature is measured by a type-K thermocouple, which is spot-welded to the back of the sample holder. The entrance window of the detector is a $2.5 \mathrm{~mm}$ thick $\mathrm{SrF}_{2}$ disc with a diameter of 1 ", which allows detection of photons of $9.5 \mathrm{eV}$ and a bandpass of approximately $0.4 \mathrm{eV}$. The detector is filled with helium to 30 Torr (uncorrected pressure read by an $\mathrm{N}_{2}$-calibrated Convectron gauge), before opening a valve to iodine crystals which are allowed to equilibrate. More details of the setup are explained in D. Tang's thesis [13], and the IPES setup and the data collecting are further discussed in B. Young's thesis [14].

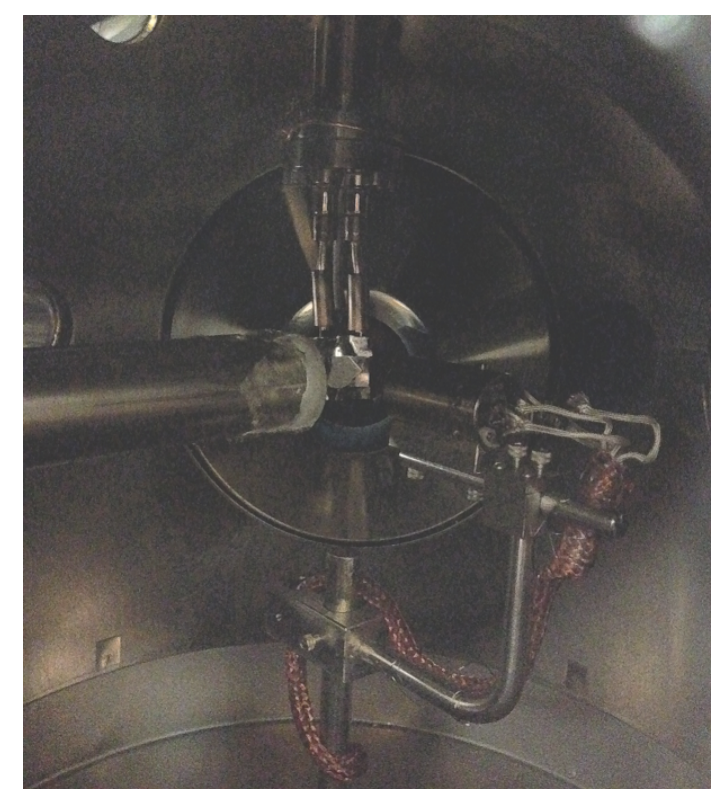

Figure 17. Sample mounted in the chamber using a sample holder, which is made with a tantalum foil folded around tungsten wires.

To generate a single inverse spectrum using IPES, the electron energy is varied from 6.5 to $10.5 \mathrm{eV}$ in steps of $0.1 \mathrm{eV}$, and each step is held for $250 \mathrm{~ms}$. 50 scans are performed to generate one spectrum. The resulting spectrum illustrates the normalized counts (normalized to the average current developed across the sample) as intensity on the y-axis versus the electron energy supplied by the electron gun on the x-axis. 
The sample co-ordinates are selected to focus on the $\bar{Y}$ point on the SBZ, and the angle of the electron incidence, $\theta$, can be obtained using equation (2). The parallel, perpendicular co-ordinates can be obtained by observation and later refined.

$$
k_{\|}=\sqrt{\frac{2 m}{\hbar^{2}}\left(\hbar \omega+E_{f}-\phi\right)} \sin (\theta)
$$

Where the final energy state is $E_{f}$, $\phi$ is the work function, for Nickel it is $5.01 \mathrm{eV}$, emitted photon energy, $\hbar \omega$ is $9.5 \mathrm{eV}$, $m$ is the mass of electron $=9.1 \times 10^{-31} \mathrm{Kg}$, $\hbar=6.582 \times 10^{-16} \mathrm{eV}$-sec, where $1 \mathrm{eV}=1.602 \times 10^{-19} \mathrm{~J}=1.602 \times 10^{-19} \frac{\mathrm{Kg}-\mathrm{m}^{2}}{\mathrm{sec}^{2}}$ $k_{\|}$corresponds to the $\bar{Y}$ point, which is about $\sim 0.93 \AA^{-1}$ (figure 1), and corresponds to an angle of the electron incidence, $\theta$ is $\sim 45^{\circ}$. 


\section{List of References}

[1] P. D. Johnson and S. L. Hulbert, "Inverse photoemission," Rev. Sci. lnstrum, vol. 61, no. 9, pp. 2277-2288, 1990.

[2] N. V. Smith and D. P. Woodruff, "Inverse photoemission from metal surfaces," Progress in surface science, vol. 21, no. 4, pp. 295-370, 1986.

[3] W. Steinmann, "Spectroscopy of image-potential states by two-photon photoemission," Applied Physics A, vol. 49, no. 4, pp. 365-377, 1989.

[4] F. Himpsel and T. Fauster, "Probing valence states with photoemission and inverse photoemission," Journal of Vacuum Science \& Technology A, vol. 2, no. 2, pp. 815-821, 1984.

[5] P. W. Erdman and E. C. Zipf, "Lowvoltage, highcurrent electron gun," Review of Scientific Instruments, vol. 53, no. 2, pp. 225-227, 1982. [Online]. Available: http://scitation.aip.org/content/aip/journal/rsi/53/2/10.1063/1.1136932

[6] Glenn F. Knoll, Radiation Detection and Measurement. John Wiley \& Sons, Inc., 2000.

[7] R. A. Bartynski, T. Gustafsson, and P. Soven, "Observation of an unoccupied surface state on cu(110) by inverse photoemission," Phys. Rev. B, vol. 31, pp. 4745-4750, Apr 1985.

[8] A. Goldmann, M. Donath, W. Altmann, and V. Dose, "Momentum-resolved inverse photoemission study of nickel surfaces," Physical Review B, vol. 32, no. 2, pp. 837-850, July 1985.

[9] R. F. Garrett and N. V. Smith, "Unoccupied bulk, surface, and image states on $\mathrm{Ni}(001), \mathrm{Ni}(111)$, and $\mathrm{Ni}(110), "$ Physical Review B, vol. 33, no. 6, pp. 3740-3744, March 1986.

[10] Gerald Burns, Solid State Physics. Orlando, Florida: Academic Press, Inc., 1985.

[11] Andrew Zangwill, Physics at surfaces. Cambridge: Cambridge University Press, 1988.

[12] "Image of commercial low energy electron diffraction (leed) by omicron." 2015. [Online]. Available: http://www.omicron.de/en/products/leed

[13] D. Tang, "The Properties of Alkali metal and Alkali-coadsorbate Overlayers on Metal surfaces," Ph.D. dissertation, University of Rhode Island, 1993.

[14] Benjamin Young, "Electronic and Chemical Properties of Surface AND Interfacial Zones," Ph.D. dissertation, University of Rhode Island, Aug 2014. 
CHAPTER 3

\section{Results and Discussion}

This chapter presents the experimental results, and discussion followed by a summary and future work. For this study three types of analysis were performed: 1. Room temperature investigation. 2. Annealing the sample while sputtering. 3. Comparing the effect of annealing the sample while sputtering to sputtering followed by annealing the sample (as two different steps).

\subsection{Results and Discussion}

In this thesis a typical experiment involves the following steps:

- Step-1: 30 minute sputtering at $0^{\circ}$ relative to the sample normal with $500 \mathrm{eV}$ $\mathrm{Ar}+$ ions at a $5 \times 10^{-5}$ Torr pressure and an emission current of $20 \mathrm{~mA}$. This step generates a clean yet disordered surface. For sputtering the chamber is back-filled with pure argon to the desired pressure. Once the sputtering is complete the argon is evacuated using the turbo-molecular pump and the chamber is allowed to recover back to $\sim 10^{-10}$ Torr.

- Step-2: Sputtering is followed by a 5 minute anneal, where the Ni crystal is heated to approximately $1000 \mathrm{~K}$ to form a clean and smooth crystal surface.

- Step-3: LEED and IPES measurements are performed on the clean surface. A LEED image is typically taken right away, whereas the sample is allowed to cool for a few minutes (by monitoring the thermocouple reading) before an IPES measurement.

- Step-4: Later surface defects are induced depending on the requirements, such as 10 minute sputter at $5 \times 10^{-6}$ Torr, followed by LEED and IPES measurements. 


\subsection{Inverse photoemission spectroscopy (IPES) Results}

Some of the inverse spectra are analyzed in IGOR and are presented below. Figure 18 illustrates two spectra after sputtering (without annealing, result of step1 outline in the above procedure), and after annealing (step 2). In all the plots, the solid lines are the smoothed counterparts of the data shown by the markers alone. The annealed spectrum (blue line), typically referred to as the clean spectrum, exhibits a peak around $2.6 \mathrm{eV}$. This corresponds to the surface state $\left(S_{1}\right)$ shown in Figure 1. The red line (bottom line) corresponds to a spectrum after 30 minute sputtering at $5 \times 10^{-5}$ without annealing, which does not exhibit any significant peak as sputtering degraded the crystal, by creating surface vacancies. When the crystal is annealed, most or all of these defects are healed after annealing. The other peak $\sim 0.3 \mathrm{eV}$ is primarily caused by the transition to the vacant d-band [1] (B1 as shown in Figure 1).

Figure 19 shows two intermediate sputtering steps along with the clean and 30 minute sputter plots. The two steps are: 5 minute sputter at $1 \times 10^{-6}$ Torr, followed by an additional 5 minute sputter at $5 \times 10^{-6}$ Torr. The bottom plot still corresponds to 30 minute sputtering at $5 \times 10^{-5}$ without annealing.

In IGOR, the data is smoothed by choosing the "Binomial" algorithm, and a smoothing factor of 2. In all the plots, the solid lines are the smoothed counterparts of the data shown by markers alone. To compare the decrease in surface peak intensity, the area around $2.6 \mathrm{eV}(2$ and $\sim 3.2 \mathrm{eV})$ is computed after smoothing and subtracting a linear background, then the remaining curves are fitted to a Gaussian peak with a bandwidth of $0.3 \mathrm{eV}$.

Figure 20 presents another example of the decrease in the surface state intensity as a function of time. Here the sputtering performed was: $2 \frac{1}{2}$ minute $-2 \frac{1}{2}$

minute $-2 \frac{1}{2}$ minute $-2 \frac{1}{2}$ (a total of $4-2 \frac{1}{2}$ sputtering), followed by an additional 


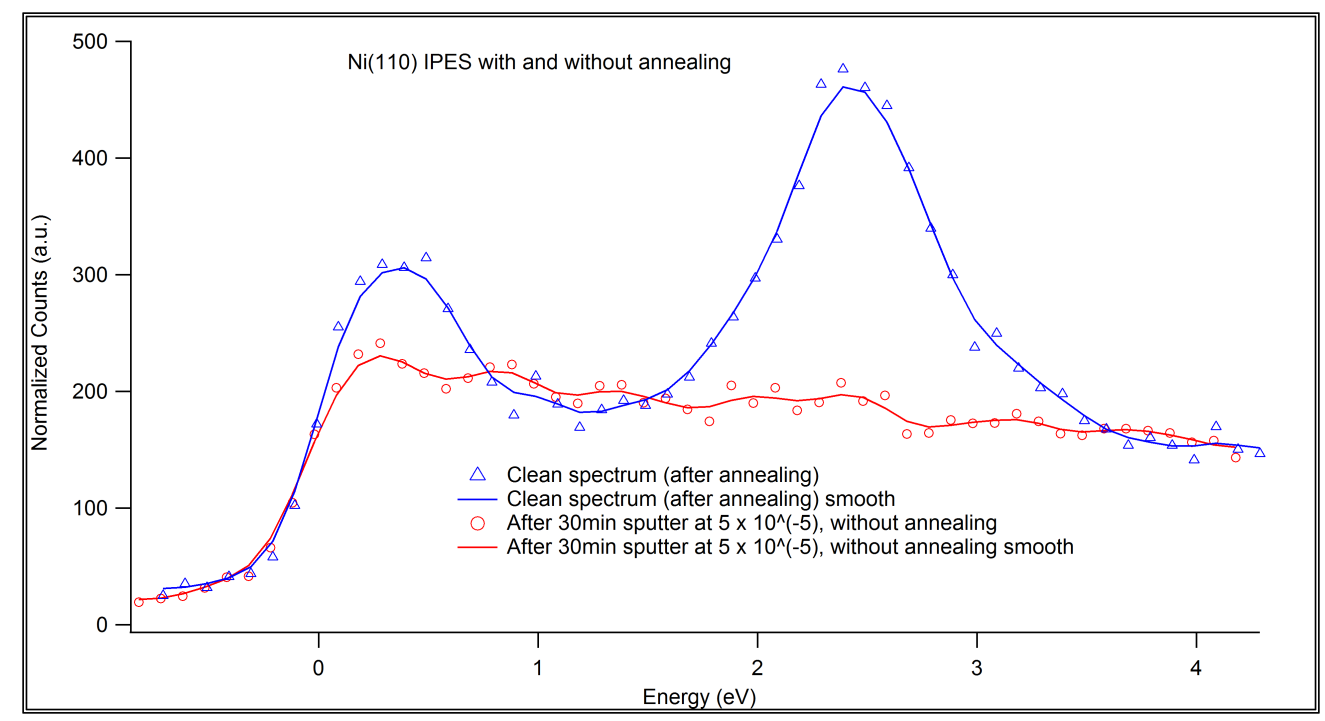

Figure 18. The plot presents an inverse photoemission spectra after sputtering (with and without annealing). The $\mathrm{x}$-axis is energy in $\mathrm{eV}$, with 0 being the the Fermi edge $E_{f}$ and normalized counts (normalized to the average current developed across the sample) on the y-axis. The annealed crystal shows a clean peak around $2.6 \mathrm{eV}$, whereas the unannealed spectrum does not exhibit any such peak. The other peak $\sim 0.3 \mathrm{eV}$ is primarily caused by transitions to the vacant d-band [1]. Also, the solid lines are the smoothed counterparts of the data shown by markers alone.

5 minute sputter at $2 \times 10^{-6}$ Torr. Again Figure 21 shows the decreasing trend. These results follow the observations made by B. Young which indicate that longer sputtering intervals decrease the surface peak intensity [2].

A plot summarizing data from Figures 19 and 20 is illustrated in Figure 21. The plots shows the ratio of the surface area with respect to the clean surface on the $\mathrm{y}$-axis; the area of the clean surface is normalized to 1 . On the $\mathrm{x}$-axis is the cumulative sputtering time. The data shows a clear decrease in the surface peak intensity with cumulative sputtering.

The cumulative time scale is computed as follows: all the sputter "times" are calculated assuming a 1 minute sputter at $5 \times 10^{-6}$ Torr. As an example, a 15 minute sputter at $2 \times 10^{-6}$ Torr can be thought as a 6 minute sputter at $5 \times 10^{-6}$ Torr. 


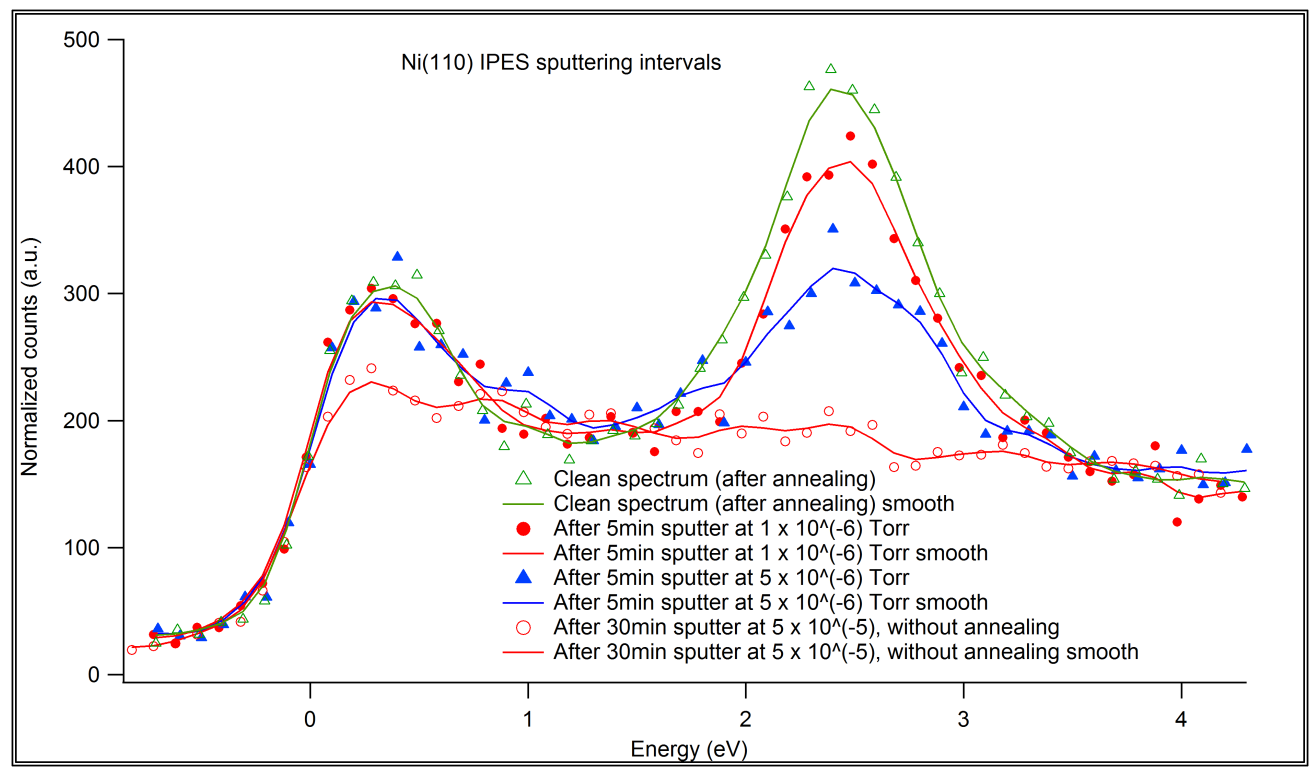

Figure 19. Inverse spectra for intermediate sputtering. The plots correspond to: clean, 5 minute sputter at $1 \times 10^{-6}$ Torr, 5 minute sputter at $5 \times 10^{-6}$ Torr, and 30 minute sputter.

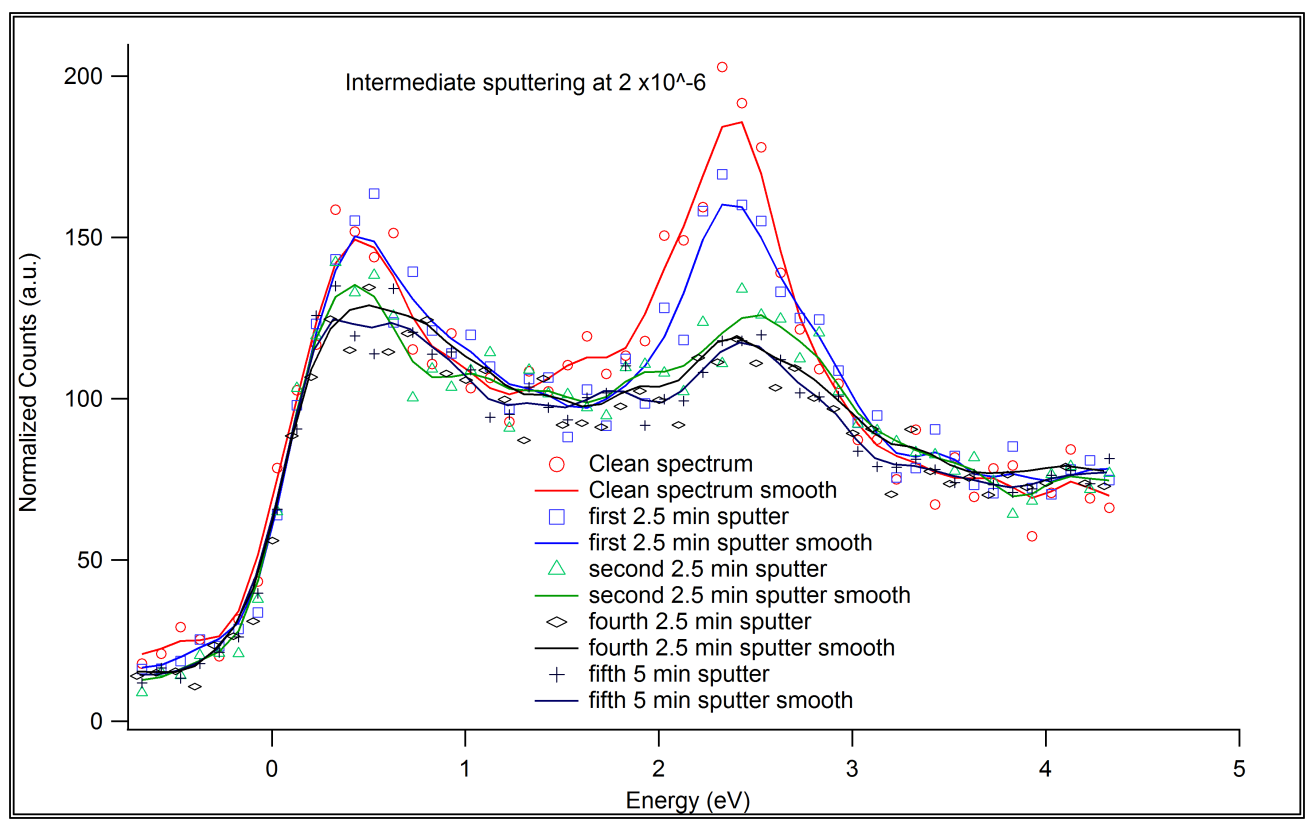

Figure 20. Inverse spectra for sputtering at 2.5 - 2.5 - 2.5 - 2. 5 - 5 minute intervals at $2 \times 10^{-6}$. The plots correspond to: clean, 2.5 minute sputter, 2.5 and 5 minute sputter. 


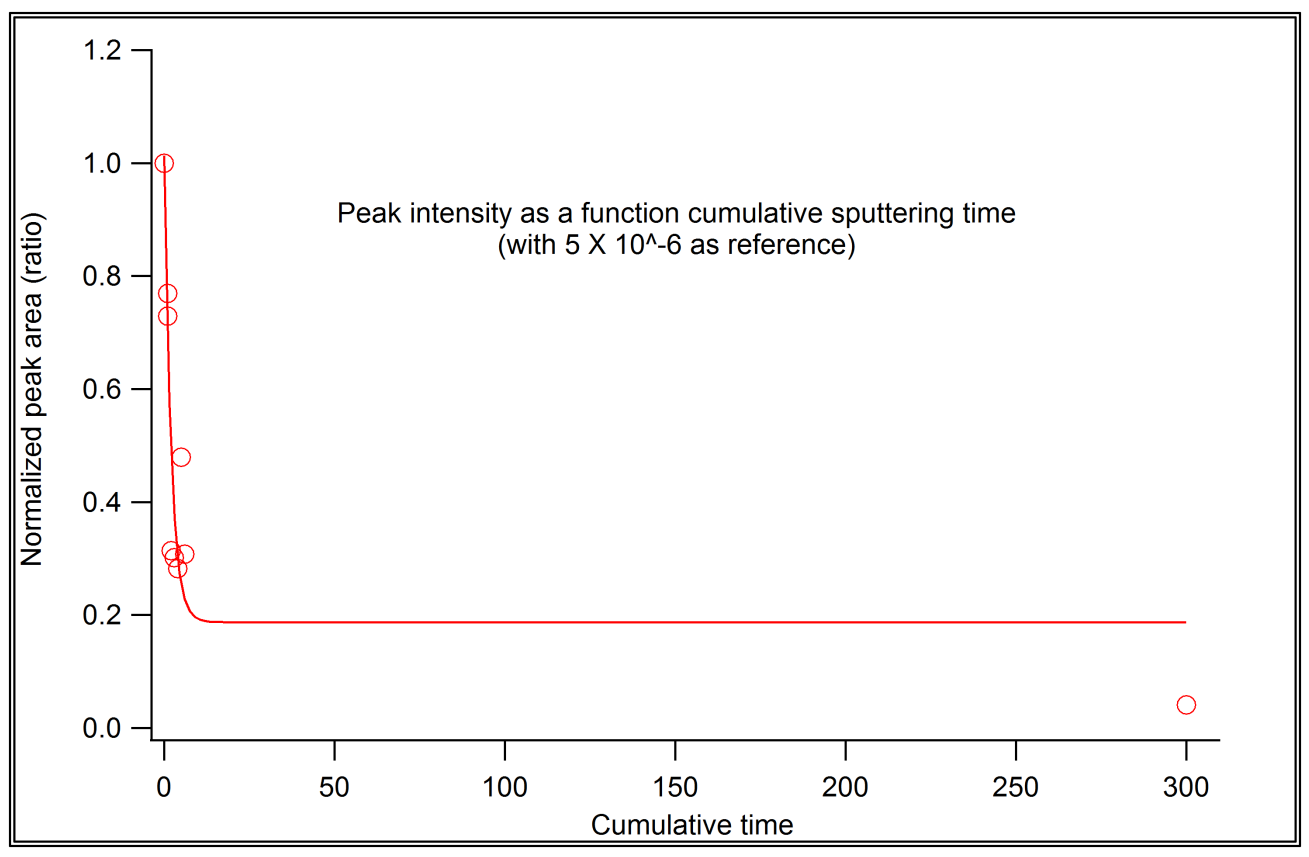

Figure 21. The plots shows the ratio of the surface area with respect to the clean surface on the y-axis; the area of the clean surface is normalized to 1 . On the x-axis is the cumulative sputtering time. The plot shows the decrease in the surface peak intensity as a function of cumulative sputter time. 
As a next area of study, the sample was annealed while sputtering. Some of the data is illustrated in Figures 22 and 23, and a few other spectra are presented in the appendix (Figures A.7 and A.8).

Figure 22 illustrates the spectra when the sample is being annealed while sputtering for 10 minutes at $5 \times 10^{-6}$ Torr. For the green (top) spectrum the sample is heated to $59{ }^{\circ} \mathrm{C}(332 \mathrm{~K})$, whereas the blue (bottom) spectrum corresponds to $35^{\circ} \mathrm{C}(308 \mathrm{~K})$. It can be observed that the surface peak $\sim 2.6 \mathrm{eV}$ is higher when the sample is annealed at a higher temperature, when compared to annealing at lower temperature.

Similar observation are illustrated in Figure 23. Here the sample is annealed at $47{ }^{\circ} \mathrm{C}(320 \mathrm{~K}), 71{ }^{\circ} \mathrm{C}(344 \mathrm{~K}), 132{ }^{\circ} \mathrm{C}(405 \mathrm{~K})$ for 10 minutes at $5 \times 10^{-6}$ Torr. The green (top) peak corresponds to $132{ }^{\circ} \mathrm{C}(405 \mathrm{~K})$, followed by $71^{\circ} \mathrm{C}(344 \mathrm{~K})$ and the red (bottom) corresponds to $47^{\circ} \mathrm{C}(320 \mathrm{~K})$. Again the surface peak is higher in intensity when the sample is annealed at a higher temperature. Supplemental data corresponding to annealing at $296.15 \mathrm{~K}, 313.15 \mathrm{~K}, 344.15 \mathrm{~K}, 325.15 \mathrm{~K}$ is illustrated in the Appendix, and all the data follows the same trend.

Figure 24 illustrates the effect of annealing while sputtering, by summarizing data from the Figures 22, 23, A.7 and A.8. The plot presents temperature on the $\mathrm{x}$-axis, and the surface peak area as a ratio with the $405 \mathrm{~K}$ peak area. When the sample was sputtered and annealed at $132{ }^{\circ} \mathrm{C}(405 \mathrm{~K})$, there is little change in the surface peak intensity. The surface peak is close to the same level as the clean spectrum. The increasing trend seems to indicate that the with annealing at higher temperature, a 10 minute sputter at $5 \times 10^{-6}$ Torr has little impact, in other words, annealing the sample at a higher temperature while sputtering has a healing effect. As anticipated, the sample heals faster at higher temperature for the same amount of sputtering. This is shown in the Figure 24 as an increase in 


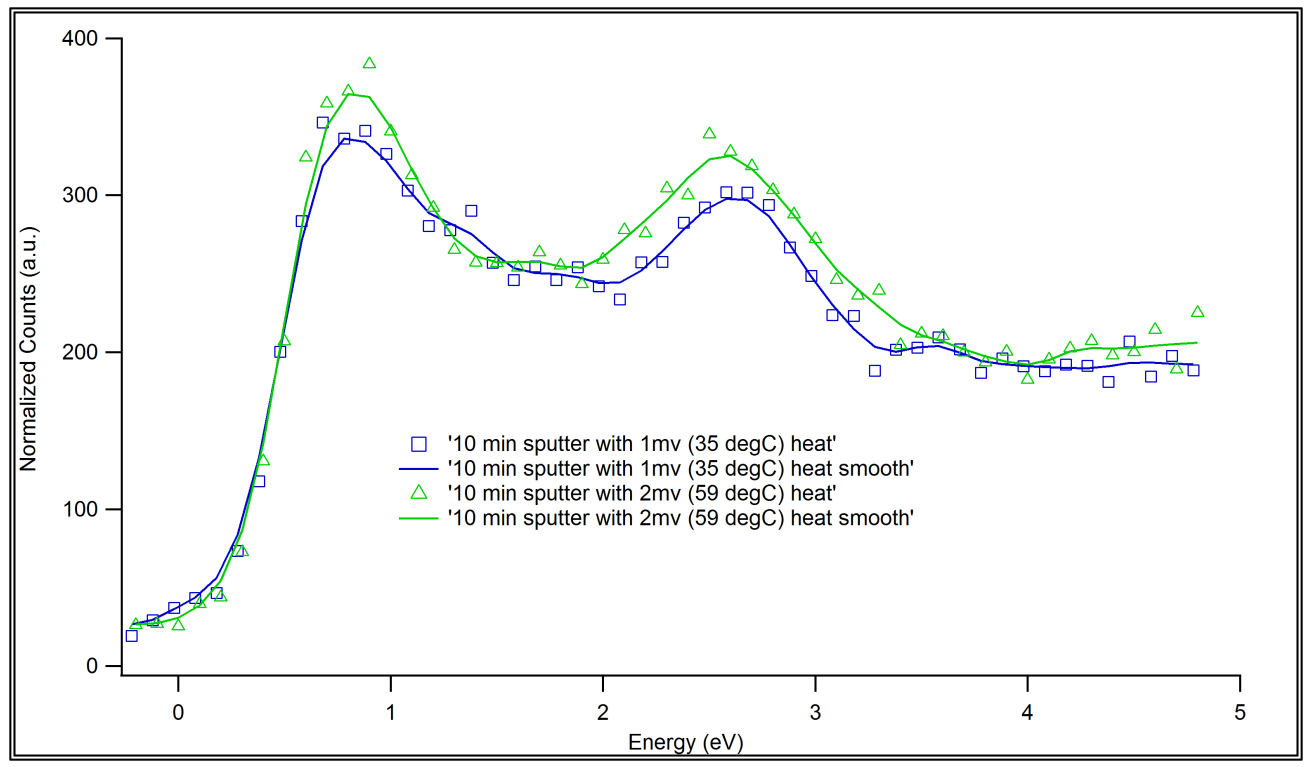

Figure 22. Inverse spectra when the sample is annealed at $35{ }^{\circ} \mathrm{C}(308 \mathrm{~K})$ and 59 ${ }^{\circ} \mathrm{C}(332 \mathrm{~K})$ while sputtering for 10 minutes at $5 \times 10^{-6}$ Torr.

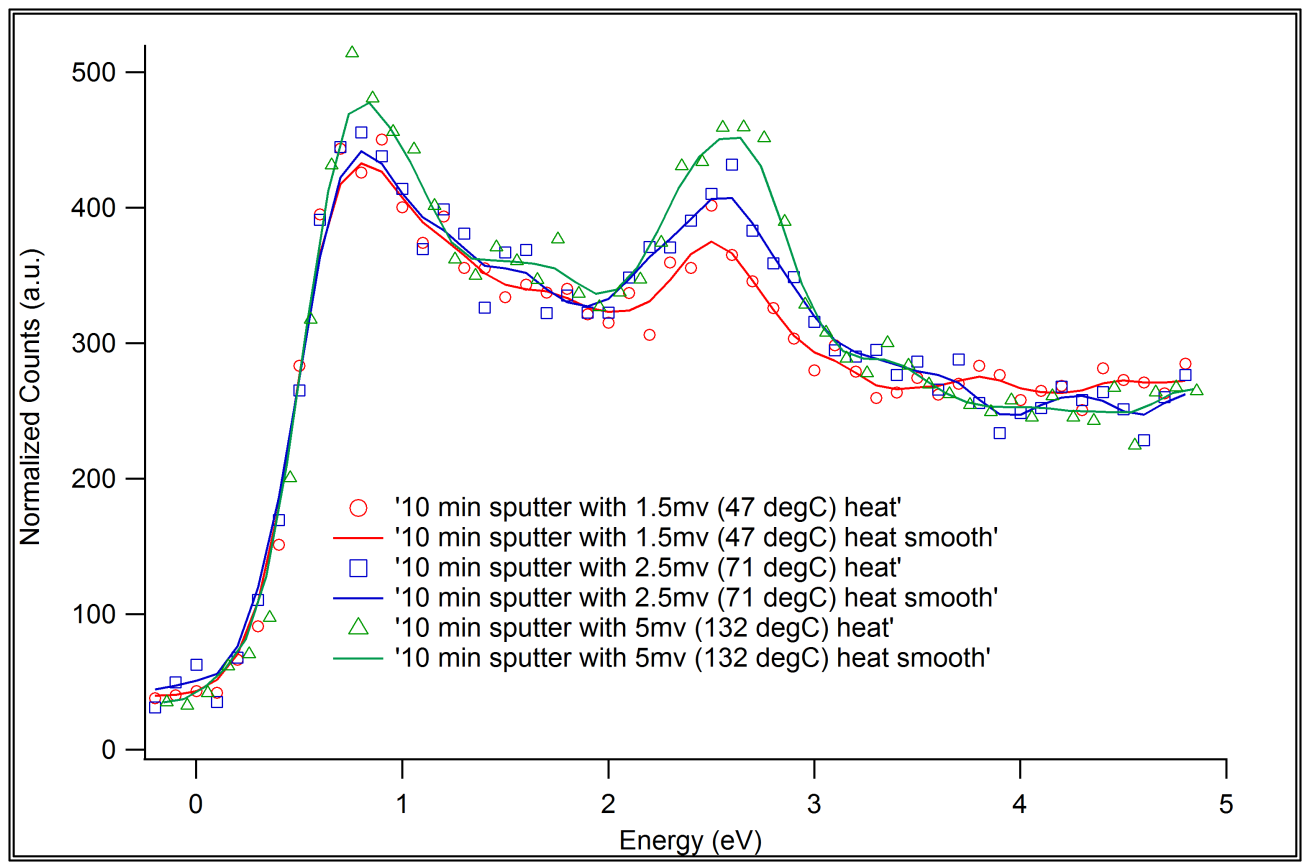

Figure 23. Inverse spectra when the sample is annealed at $47{ }^{\circ} \mathrm{C}(320 \mathrm{~K}), 71{ }^{\circ} \mathrm{C}$ $(344 \mathrm{~K}), 132{ }^{\circ} \mathrm{C}(405 \mathrm{~K})$ for 10 minutes at $5 \times 10^{-6}$ Torr.

the intensity of the surface peak as a function of increasing temperature. There are a few outliers, however the trend seems to agree with the statement. 


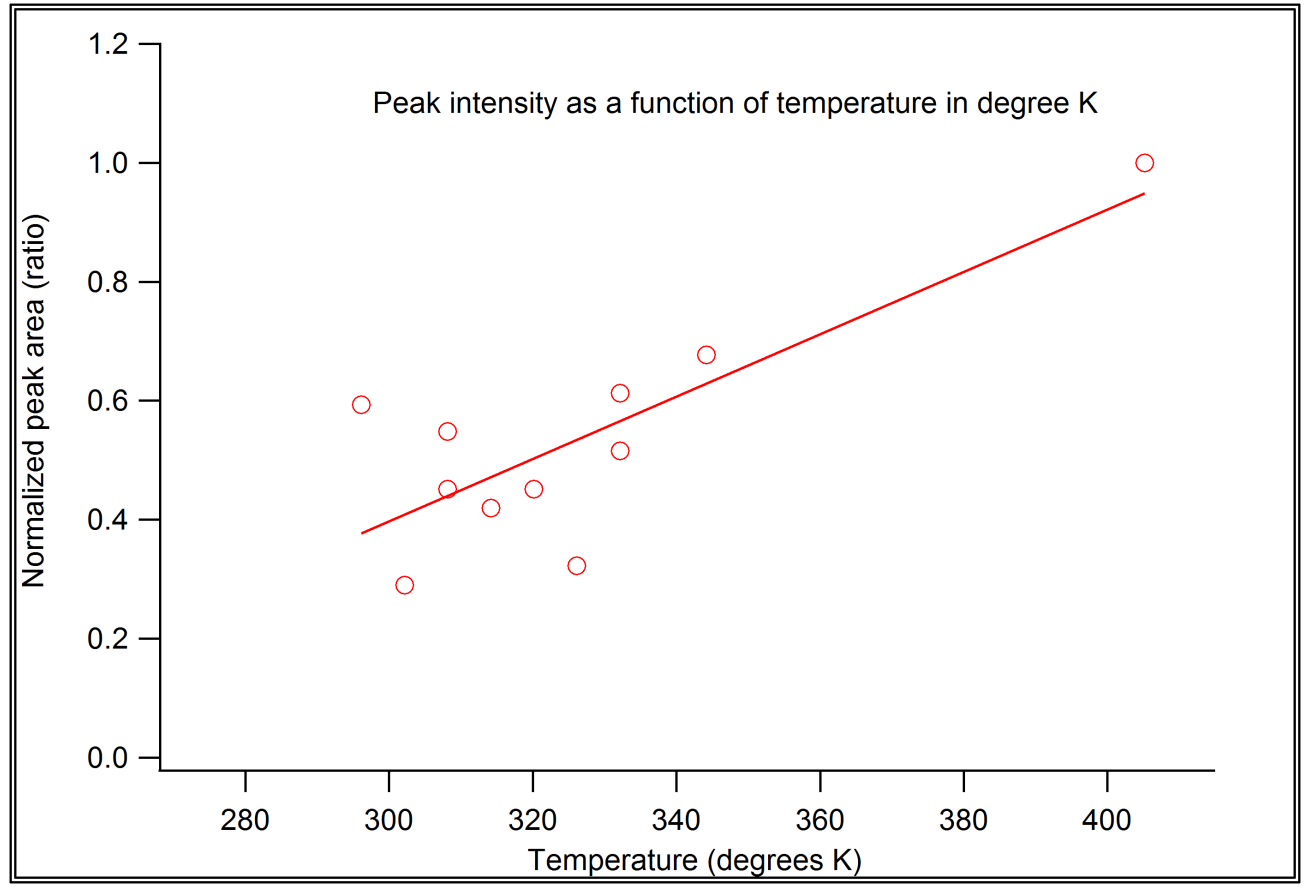

Figure 24. The plot presents temperature on the $\mathrm{x}$-axis, and the ratio of the surface peak intensity with respect to $132{ }^{\circ} \mathrm{C}(405 \mathrm{~K})$ for a 10 minute sputter at $5 \times 10^{-6}$ Torr. 
As a third analysis, a comparison between (1) annealing the sample while sputtering for 10 minutes at $5 \times 10^{-6}$ Torr and (2) a 10 minute anneal after a 10 minute sputter at $5 \times 10^{-6}$ Torr was carried out. For this case, Figure 25 illustrates the inverse spectra at $35^{\circ} \mathrm{C}(308 \mathrm{~K})$. Similarly Figures 26 and 27 illustrate the inverse spectra for $23^{\circ} \mathrm{C}(296 \mathrm{~K})$ and $29{ }^{\circ} \mathrm{C}(302 \mathrm{~K})$ temperatures respectively. There seems to be some difference when the sample was prepared at $35^{\circ} \mathrm{C}(308$ K) as illustrated in Figure 25. The peak is higher for annealing while sputtering versus annealing after sputtering. However at $23^{\circ} \mathrm{C}(296 \mathrm{~K})$ and $29{ }^{\circ} \mathrm{C}(302 \mathrm{~K})$ the Ni crystal did not show any difference in the surface peak intensity (area).

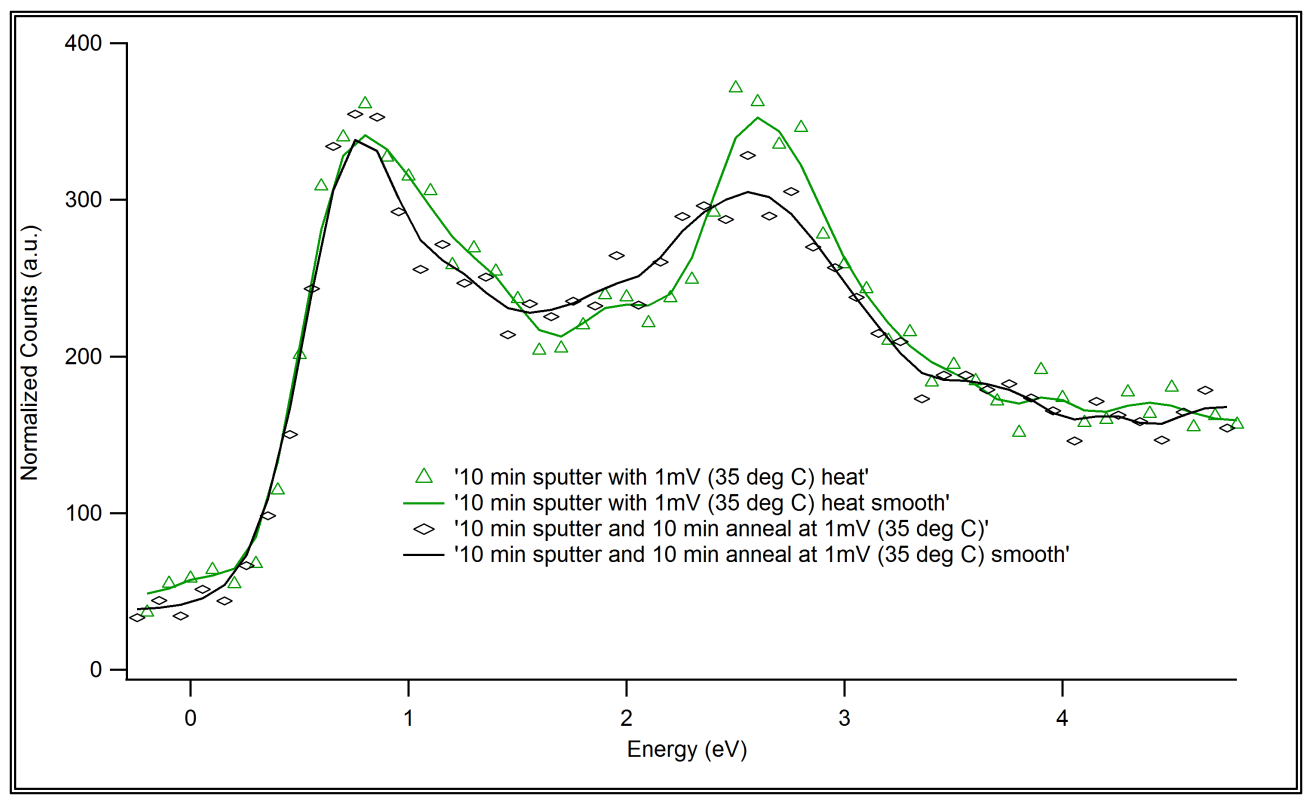

Figure 25. Inverse spectra when the sample is annealed at $35^{\circ} \mathrm{C}(308 \mathrm{~K})$ during a 10 minute sputter at $5 \times 10^{-6}$ Torr, compared to a 10 minute sputter at $5 \times 10^{-6}$ Torr followed by a 10 minute anneal at $35^{\circ} \mathrm{C}(308 \mathrm{~K})$. 


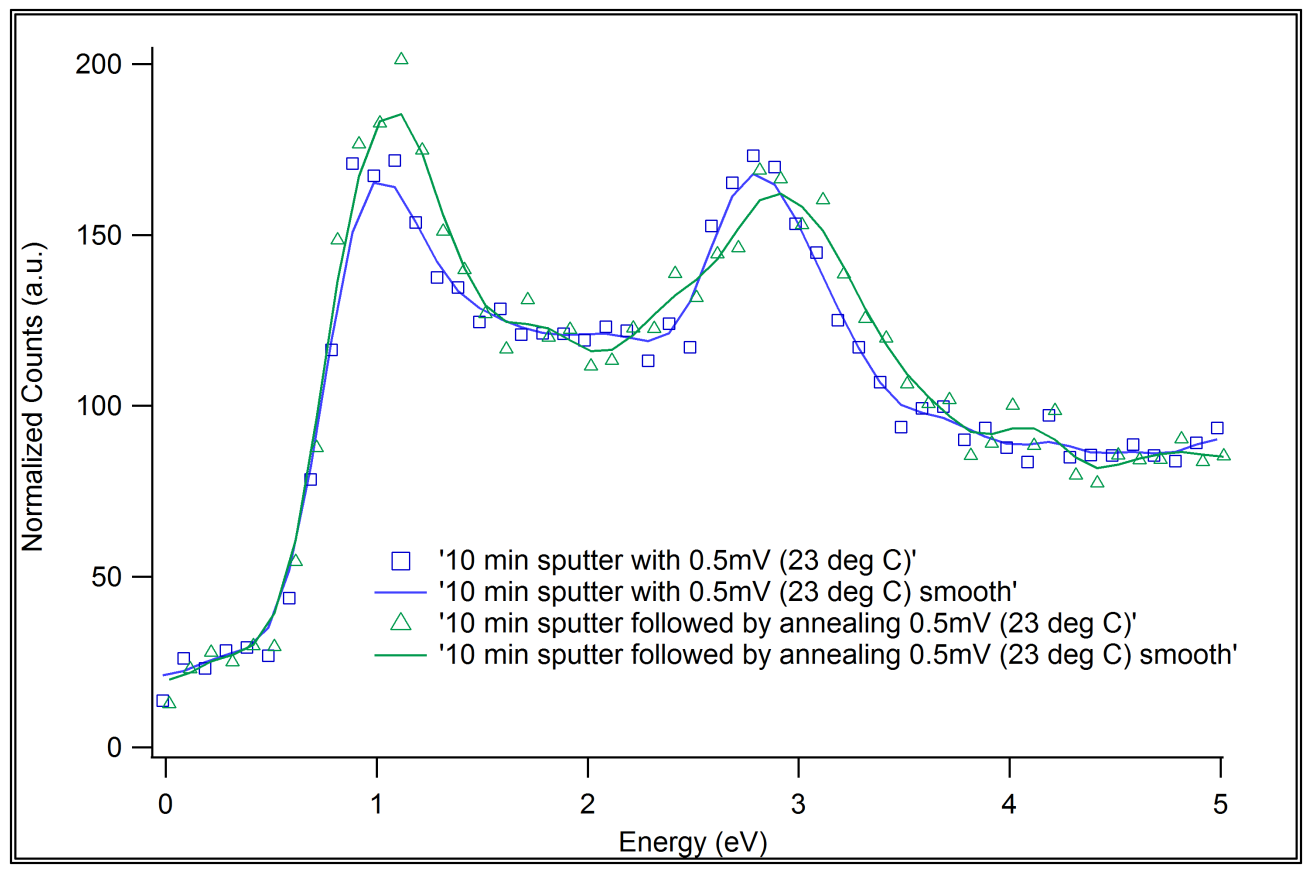

Figure 26. Inverse spectra when the sample is annealed at $23^{\circ} \mathrm{C}(296 \mathrm{~K})$, during a 10 minute sputter at $5 \times 10^{-6}$ Torr, compared to a 10 minute sputter at $5 \times 10^{-6}$ Torr followed by a 10 minute anneal at $23^{\circ} \mathrm{C}(296 \mathrm{~K})$.

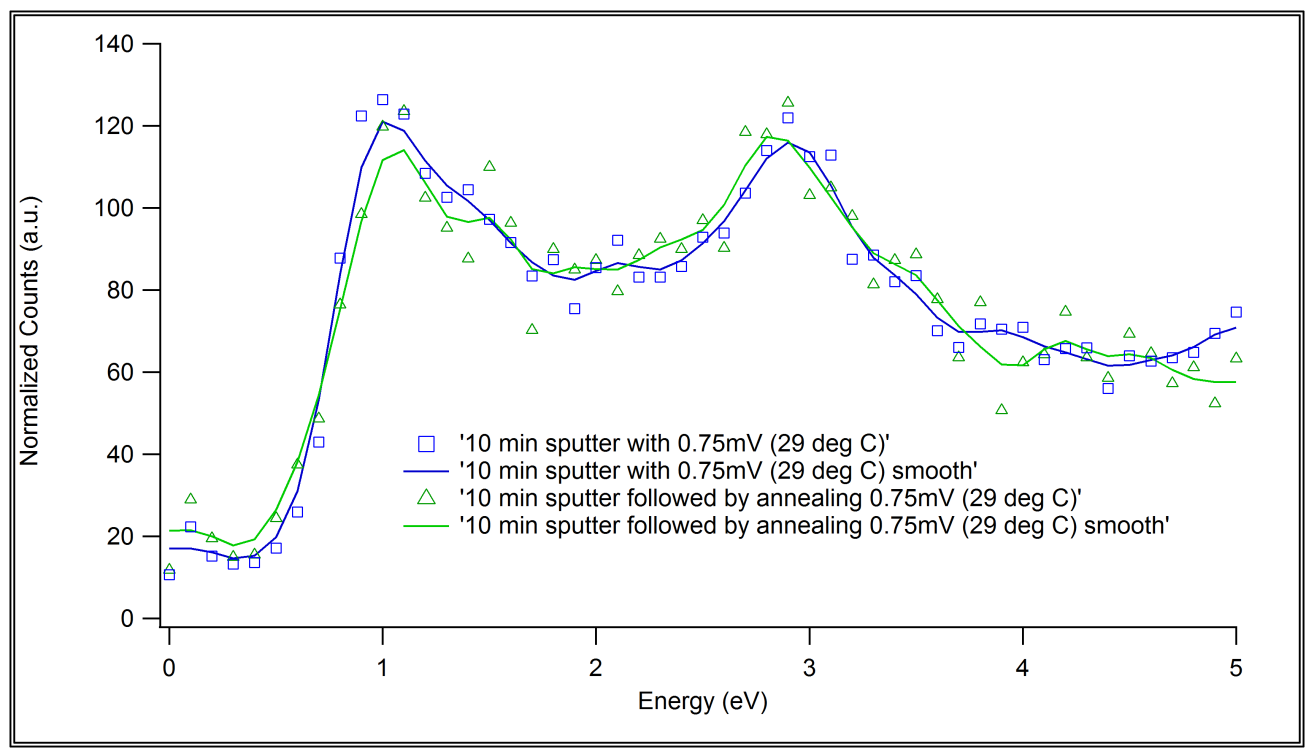

Figure 27. Inverse spectra when the sample is annealed at $29{ }^{\circ} \mathrm{C}(302 \mathrm{~K})$, during a 10 minute sputter at $5 \times 10^{-6}$ Torr, compared to a 10 minute sputter at $5 \times 10^{-6}$ Torr followed by a 10 minute anneal at $29{ }^{\circ} \mathrm{C}(302 \mathrm{~K})$. 


\subsection{LEED results}

For all LEED measurements, a $120 \mathrm{eV}$ electron beam energy and emission current of $1 \mathrm{~mA}$ was used. The phosphor screen voltage was set to $5 \mathrm{keV}$. LEED images were acquired with a home-built CCD camera located on the opposite side of the LEED phosphor screen.

Figures 28 to 30 show the LEED patterns for: (1) sputtered for 30 minutes. (2) after 5 minutes of annealing to $1000 \mathrm{~K}$. (3) after a 10 minute sputter at 5 $\times 10^{-6}$ Torr. (4) 10 minute sputter at $5 \times 10^{-6}$ Torr while annealing at $35^{\circ} \mathrm{C}(308$ K). (5) after a 10 minute sputter at $5 \times 10^{-6}$ Torr followed by a 10 min anneal at $35^{\circ} \mathrm{C}(308 \mathrm{~K})$. The annealed surface does show a clearer spot pattern compared to a surface without annealing and a sputtered surface.

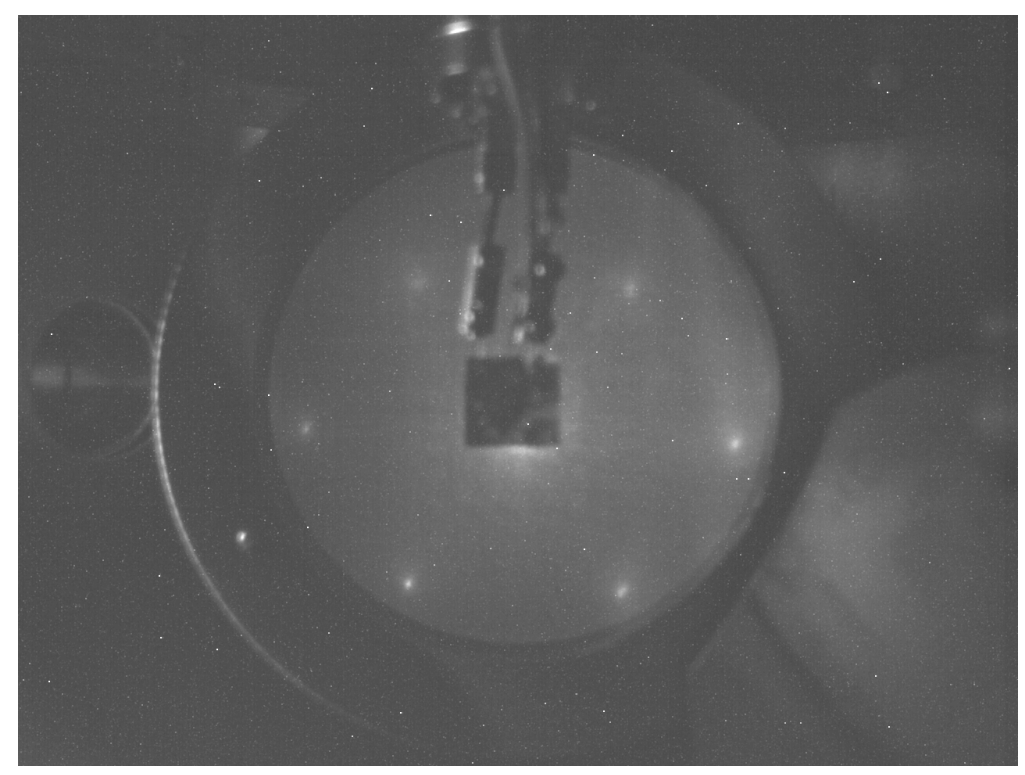

Figure 28. LEED image without annealing after a 30 minute sputter at $5 \times 10^{-5}$ Torr. 


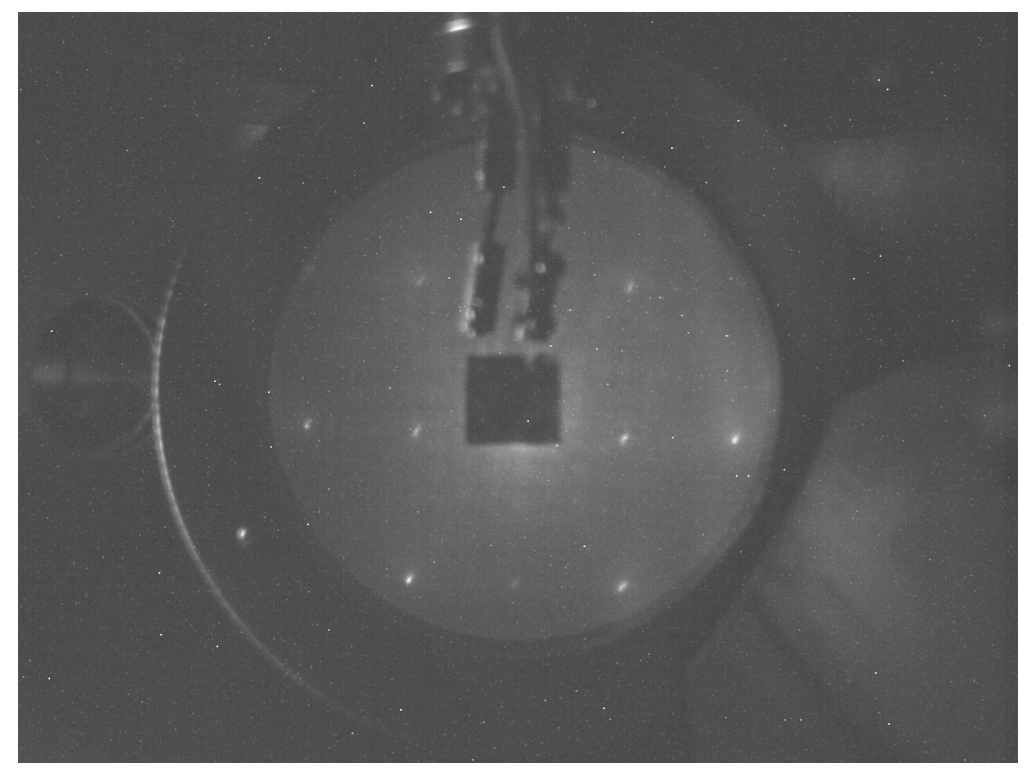

Figure 29. LEED image after a 5 minute annealing.

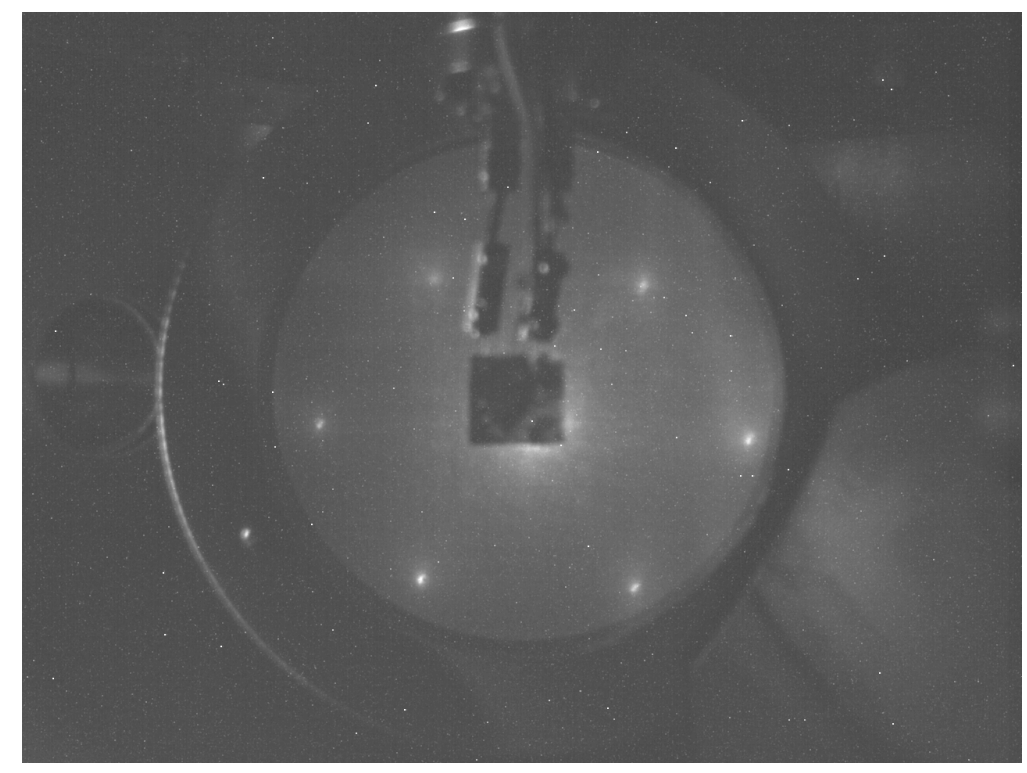

Figure 30. LEED image after a 10 minute sputter at $5 \times 10^{-6}$ Torr. 


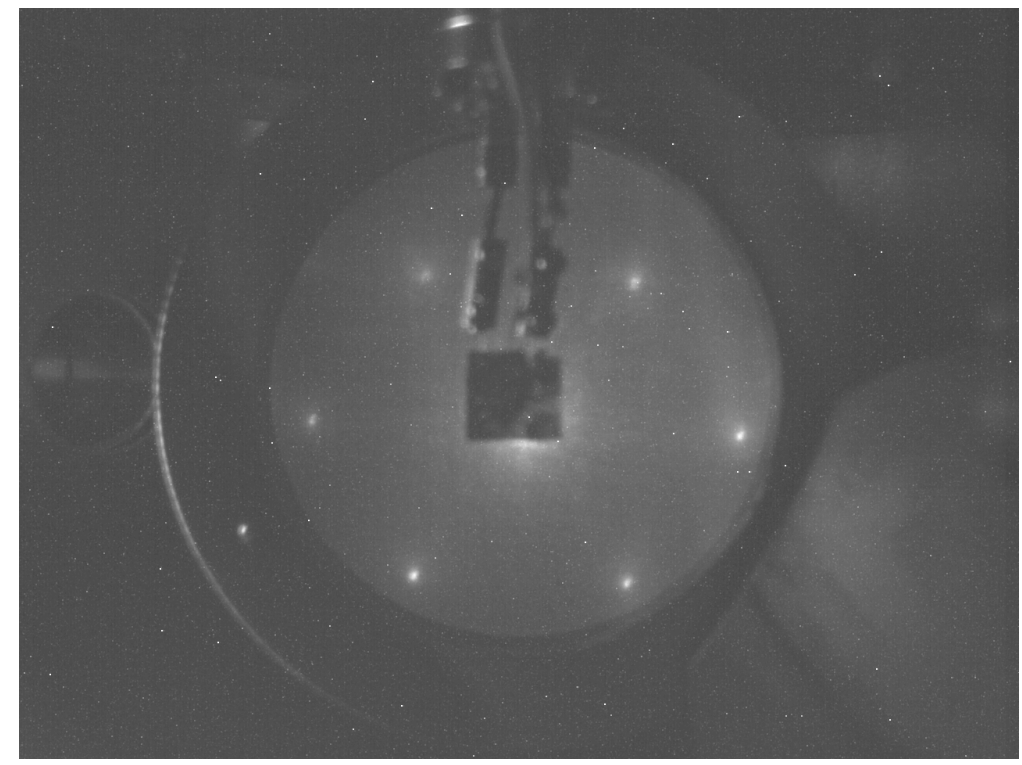

Figure 31. LEED image after a 10 minute sputter at $5 \times 10^{-6}$ Torr while annealing at at $35^{\circ} \mathrm{C}(308 \mathrm{~K})$.

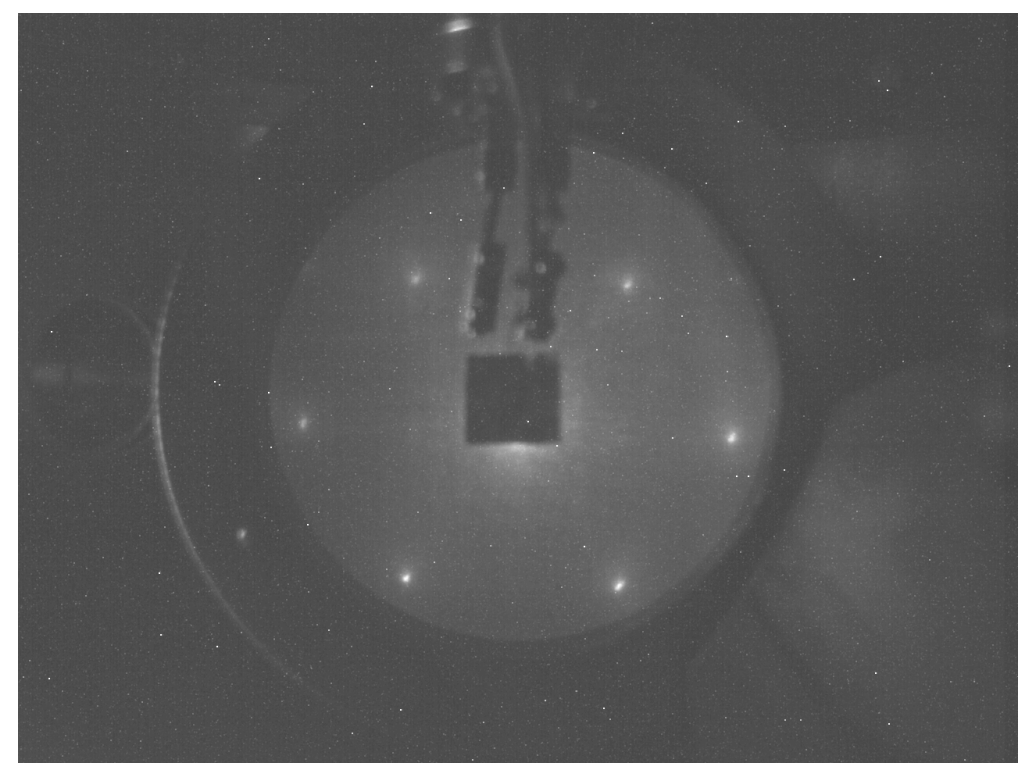

Figure 32. LEED image after a 10 minute sputter at $5 \times 10^{-6}$ Torr followed by a 10 min anneal at $35^{\circ} \mathrm{C}(308 \mathrm{~K})$. 


\subsection{Summary and Future work}

The behavior of ion-induced defects on the Ni(110) surface at elevated temperatures was examined using inverse photoemission spectroscopy and low energy electron diffraction.

The clean Ni (110) surface exhibits a peak $\sim 2.6 \mathrm{eV}$ above the Fermi level, indicating an unoccupied surface state near the $\bar{Y}$ point of the surface Brillouin zone (SBZ). Defects are induced by low energy ion bombardment at various temperatures, which result in a decrease of the peak intensity. The surface state eventually disappears when bombarded for longer times. We also observed that the surface heals faster when the crystal is being simultaneously sputtered and annealed at higher versus lower temperature. Finally the data for annealing while sputtering versus annealing after sputtering does not seem to exhibit much difference.

The next step would be to model a Monte Carlo based simulation of the IPES data to draw further observations. From the data presented for annealing while sputtering and annealing after sputtering for the same conditions doesn't seem to have a major effect. However more data is required.

The spots from the LEED data show some qualitative differences due to annealing after ion bombardment, however to get a better idea of temperature dependence a quantitative study is necessary. This includes digitizing the LEED spots and determine their intensities.

\section{List of References}

[1] A. Goldmann, M. Donath, W. Altmann, and V. Dose, "Momentum-resolved inverse photoemission study of nickel surfaces," Physical Review B, vol. 32, no. 2, pp. 837-850, July 1985.

[2] Benjamin Young, "Electronic and Chemical Properties of Surface AND Interfacial Zones," Ph.D. dissertation, University of Rhode Island, Aug 2014. 


\section{APPENDIX}

\section{Additional reference material}

In this appendix supplemental material is included.

\section{A.1 Ni sputtering result at 170 and $300 \mathrm{~K}$ [1]}
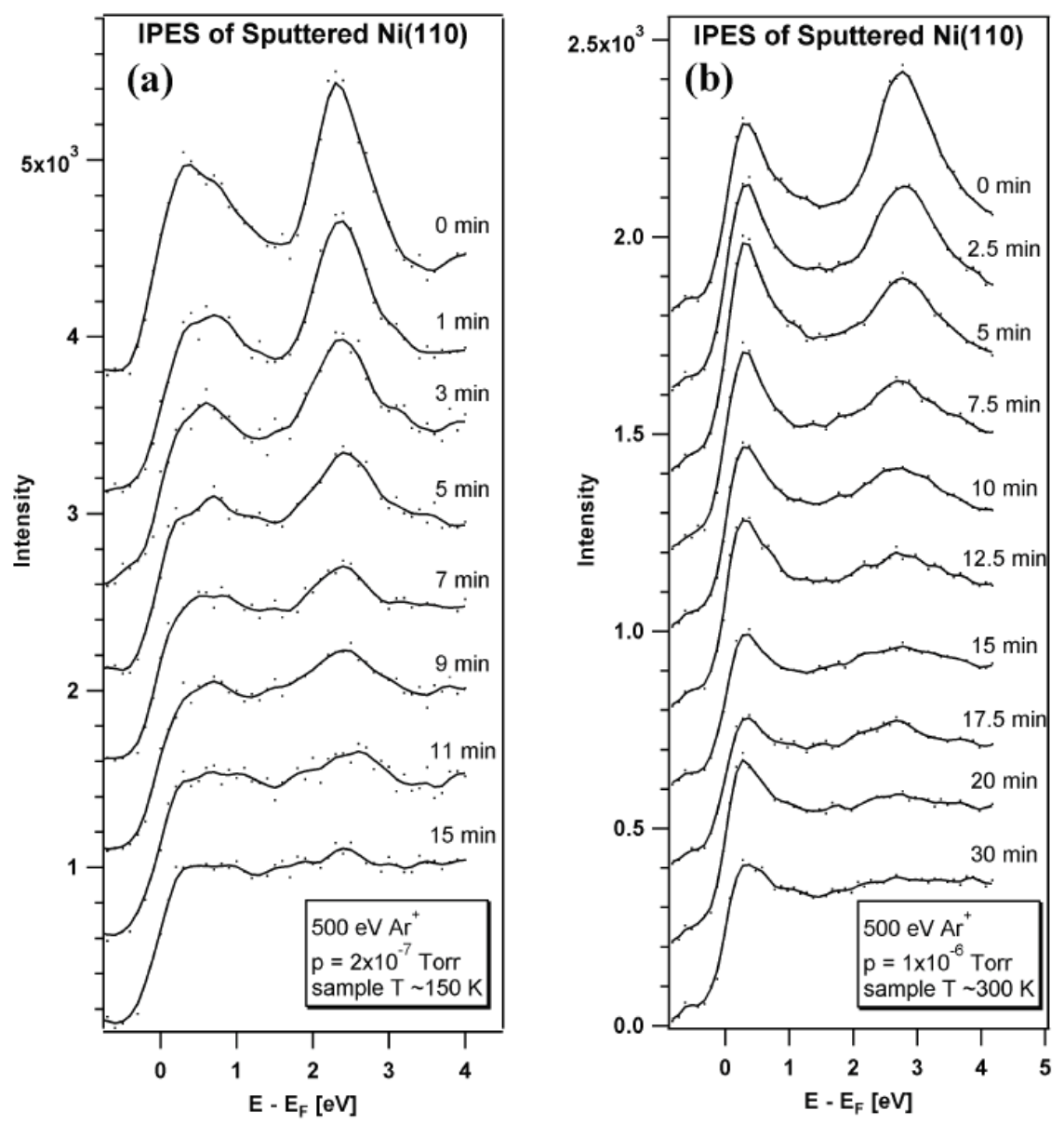

Figure A.1. B. Young's Ni (110) results for 170 and 300 K [1] 
A.2 Face centered cubic (FCC) lattice
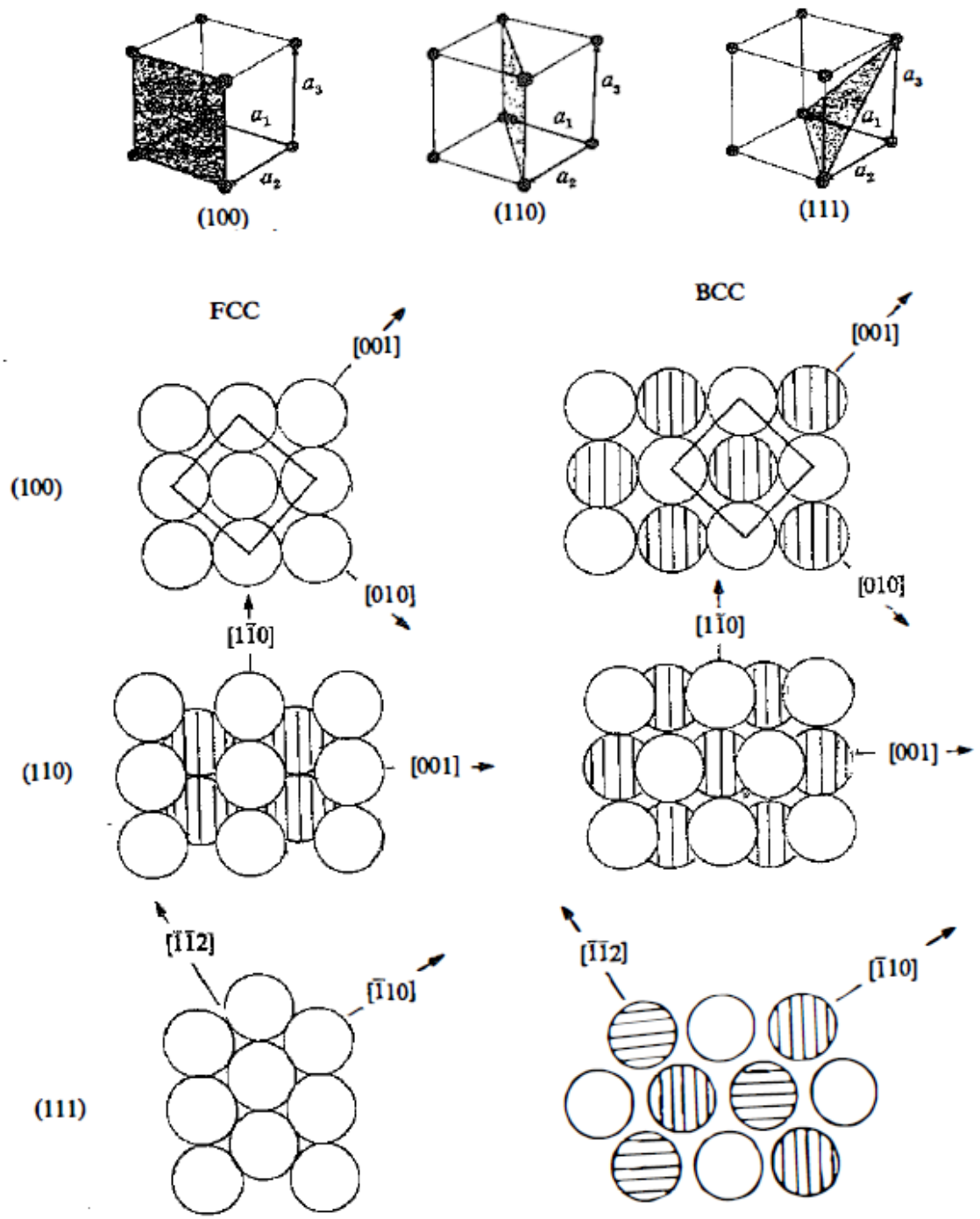

Figure A.2. FCC lattice showing the (100), (110), (111) faces and unit cells [2] 


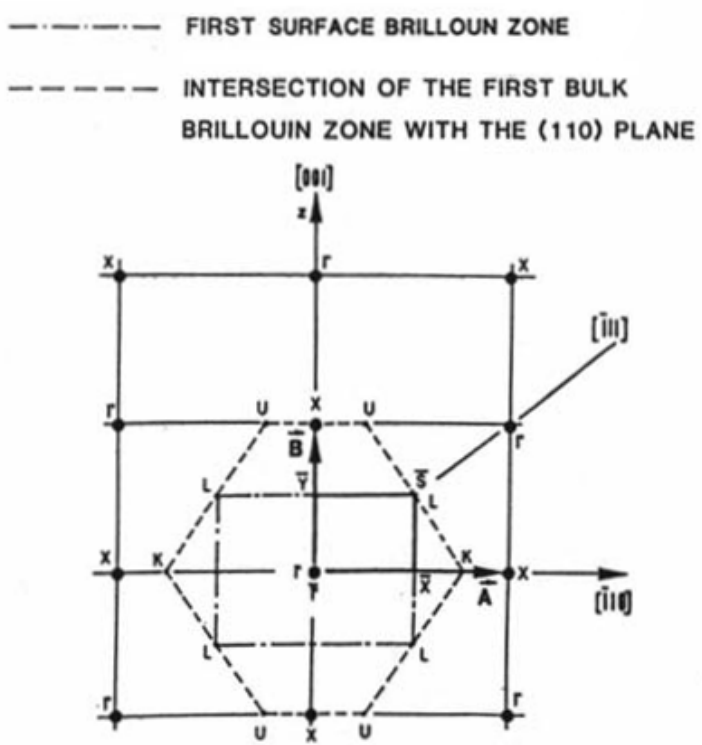

Figure A.3. Ni (110) surface brillouin zone (SBZ) projection to the bulk brillouin zone [3]

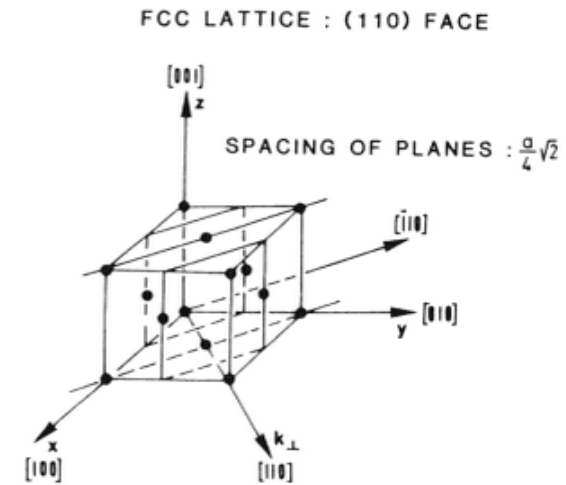

UNIT CELL OF THE (110)FACE

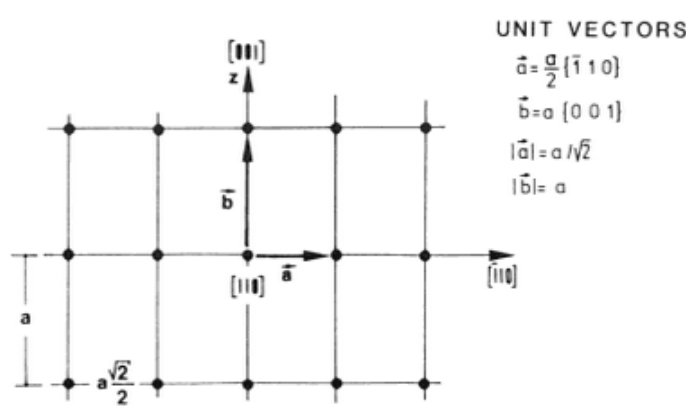

Figure A.4. FCC lattice showing the 110 face and unit cell [3] 


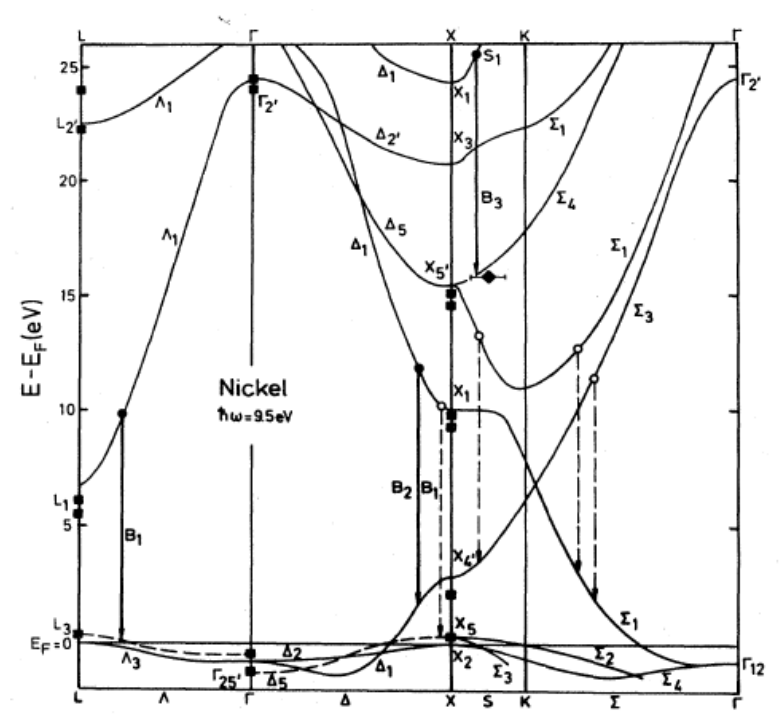

Figure A.5. Energy versus momentum band diagram for Nickel [4]

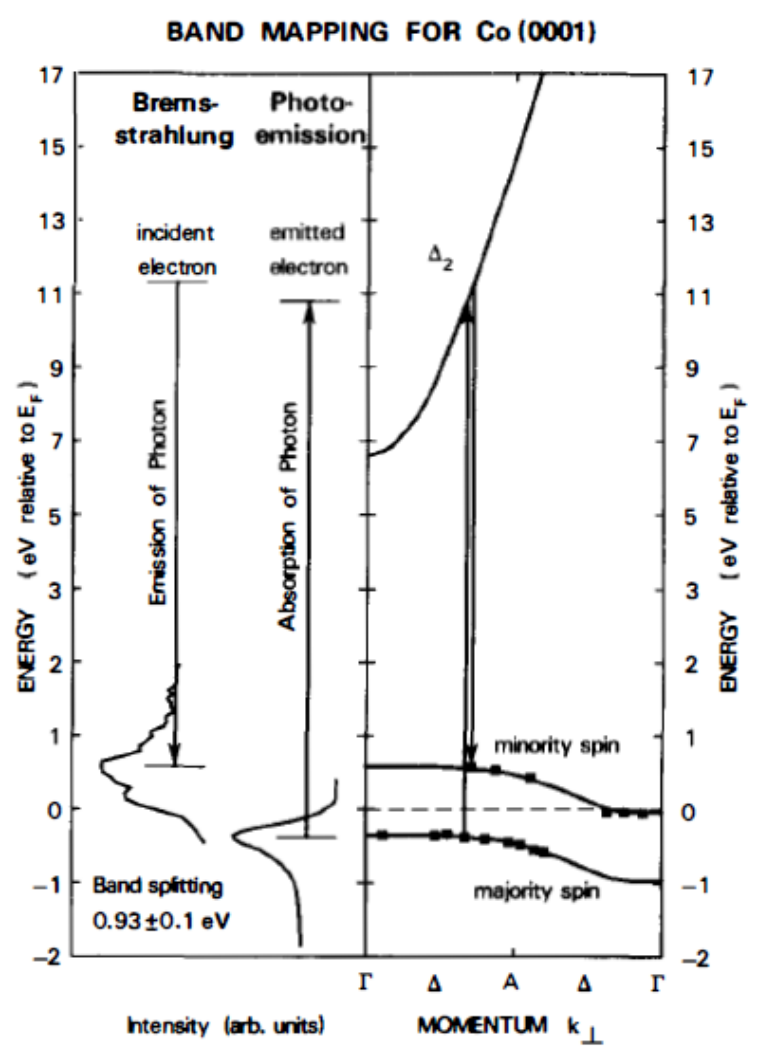

Figure A.6. Band mapping of photoemission (PES) versus inverse photoemission (IPES) for Co(0001) sample (Cobalt). For the same conditions, in IPES shows the unoccupied states corresponding to the minority $3 \mathrm{~d}$ bands, whereas the PES provides information about the majority spin states [5]. 


\section{A.3 Other IPES results}

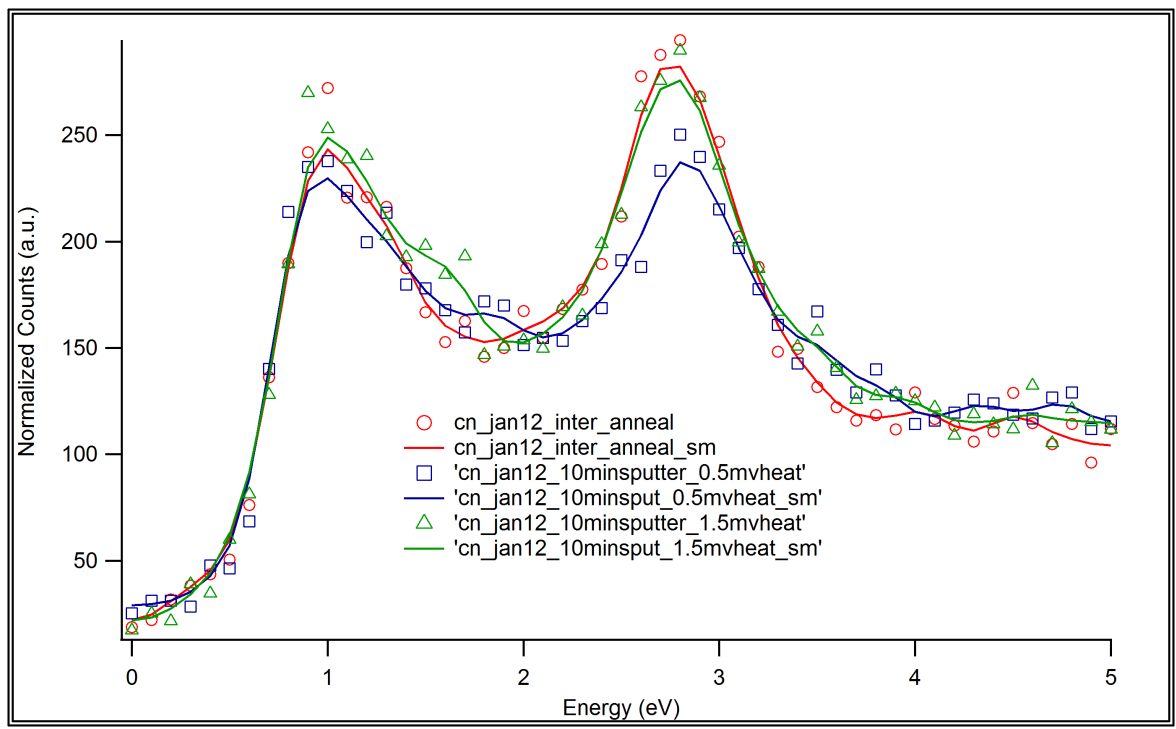

Figure A.7. Inverse spectra when the sample is annealed at $23{ }^{\circ} \mathrm{C}(396 \mathrm{~K})$ and 40 ${ }^{\circ} \mathrm{C}(413 \mathrm{~K})$ for 10 minutes at $5 \times 10^{-6}$ Torr.

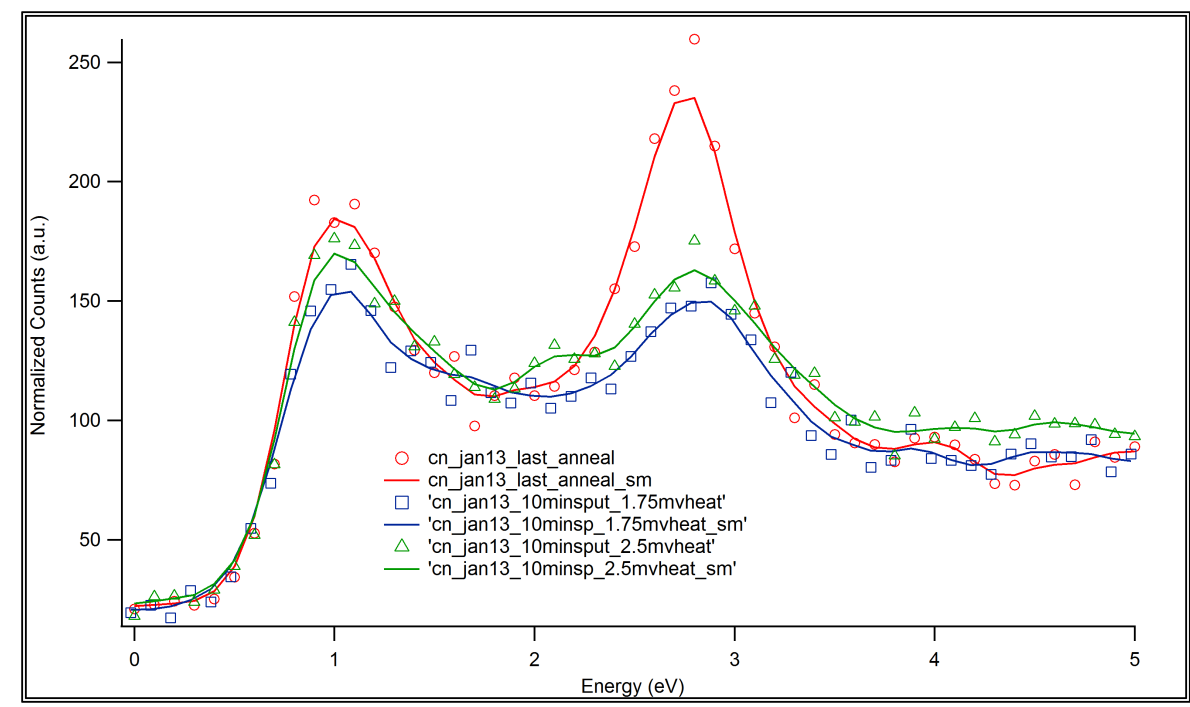

Figure A.8. Inverse spectra when the sample is annealed at $71^{\circ} \mathrm{C}(444 \mathrm{~K})$ and 52 ${ }^{\circ} \mathrm{C}(425 \mathrm{~K})$ for 10 minutes at $5 \times 10^{-6}$ Torr. 


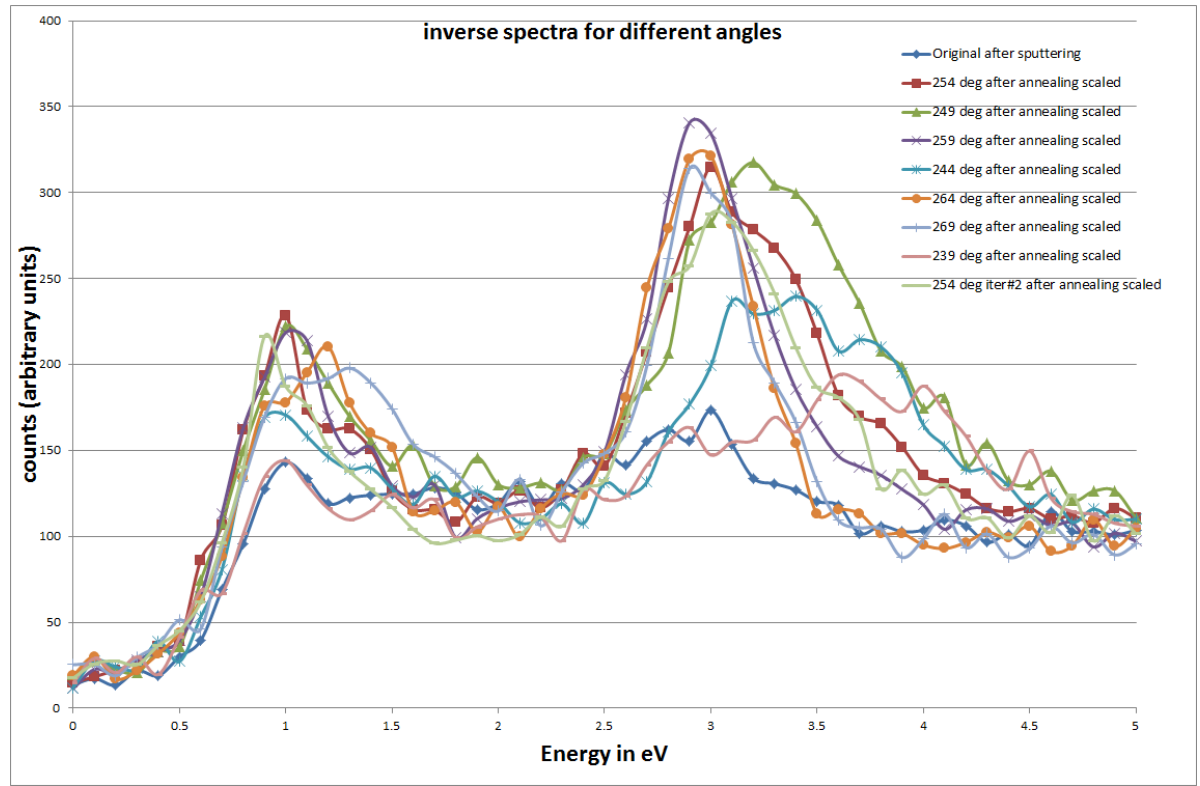

Figure A.9. inverse spectra for different detection angles

\section{List of References}

[1] Benjamin Young, "Electronic and Chemical Properties of Surface AND Interfacial Zones," Ph.D. dissertation, University of Rhode Island, Aug 2014.

[2] Andrew Zangwill, Physics at surfaces. Cambridge: Cambridge University Press, 1988.

[3] Stephan Hüfner, Photoelectron Spectroscopy Principles and Applications. Springer, 2003.

[4] A. Goldmann, M. Donath, W. Altmann, and V. Dose, "Momentum-resolved inverse photoemission study of nickel surfaces," Physical Review B, vol. 32, no. 2, pp. 837-850, July 1985.

[5] F. Himpsel and T. Fauster, "Probing valence states with photoemission and inverse photoemission," Journal of Vacuum Science \& Technology A, vol. 2, no. 2, pp. 815-821, 1984. 


\section{BIBLIOGRAPHY}

"Image of commercial low energy electron diffraction (leed) by omicron." 2015. [Online]. Available: http://www.omicron.de/en/products/leed

A. Goldmann, M. Donath, W. Altmann, and V. Dose, "Momentum-resolved inverse photoemission study of nickel surfaces," Physical Review B, vol. 32, no. 2, pp. 837-850, July 1985.

A. Goldmann, V. Dose and G. Borstel, "Empty electronic states at the (100), (110), and (111) surfaces of nickel, copper, and silver," Physical Review B, vol. 32, no. 4, pp. 971-1980, August 1985.

Andrew Zangwill, Physics at surfaces. Cambridge: Cambridge University Press, 1988.

Bartynski, R. A., Gustafsson, T., and Soven, P., "Observation of an unoccupied surface state on $\mathrm{cu}(110)$ by inverse photoemission," Phys. Rev. B, vol. 31, pp. 4745-4750, Apr 1985.

Benjamin Young, "Electronic and Chemical Properties of Surface AND Interfacial Zones," Ph.D. dissertation, University of Rhode Island, Aug 2014.

D. Heskett, D. DePietro, G. Sabatino, M. Tammaro, "Ion bombardment-induced changes in the electronic structure of $\mathrm{Cu}(110)$ investigated with inverse photoemission and computer simulations," Surface Science, vol. 513, pp. 405-411, 2002

D. P. Woodruff, "Solved and unsolved problems in surface structure determination," Surface Science, pp. 147-171, 2002.

D. Tang, "The Properties of Alkali metal and Alkali-coadsorbate Overlayers on Metal surfaces," Ph.D. dissertation, University of Rhode Island, 1993.

D. Tang and D. Heskett, "Unoccupied electronic structure of Na/Ni(111)," Physical Review B, vol. 47, no. 16, pp. 695-699, 1993.

Erdman, P. W. and Zipf, E. C., "Lowvoltage, highcurrent electron gun," Review of Scientific Instruments, vol. 53, no. 2, pp. 225-227, 1982. [Online]. Available: http://scitation.aip.org/content/aip/journal/rsi/53/2/10.1063/1.1136932

F. J. Himpsel, J. A. Knapp, and D. E. Eastman, "Experimental energy-band dispersions and exchange splitting for Ni," Physical Review B, vol. 19, no. 6, pp. 2919-2927, 1979.

Gerald Burns, Solid State Physics. Orlando, Florida: Academic Press, Inc., 1985. 
Glenn F. Knoll, Radiation Detection and Measurement. John Wiley \& Sons, Inc., 2000.

Himpsel, F. and Fauster, T., "Probing valence states with photoemission and inverse photoemission," Journal of Vacuum Science 86 Technology A, vol. 2, no. 2, pp. 815-821, 1984.

J. J. Bellina and H. E. Farnsworth, "Ion Bombardment Induced Surface Damage in Tungsten and Molybdenum Single Crystals," The Journal of Vacuum Science and Technology, vol. 9, no. 2, pp. 616-619, 1972.

M. A. Vasylyev, A. G. Blaschuk, N. S. Mashovets and N. Yu. Vilkova, "LEED study of $\mathrm{Ni}(100)$ and (111) surface damage caused by Ar+ ion bombardment with low energy and small doses," Vacuum, vol. 57, pp. 71-80, 2000.

N. V. Smith and D. P. Woodruff, "Inverse photoemission from metal surfaces," Progress in surface science, vol. 21, no. 4, pp. 295-370, 1986.

P. D. Johnson and S. L. Hulbert, "Inverse photoemission," Rev. Sci. lnstrum, vol. 61, no. 9, pp. 2277-2288, 1990.

R. F. Garrett and N. V. Smith, "Unoccupied bulk, surface, and image states on Ni(001), Ni(111), and Ni(110)," Physical Review B, vol. 33, no. 6, pp. 37403744, March 1986.

Smith, N. V., "Phase analysis of image states and surface states associated with nearly-free-electron band gaps," Phys. Rev. B, vol. 32, pp. 3549-3555, Sep 1985. [Online]. Available: http://link.aps.org/doi/10.1103/PhysRevB.32.3549

Steinmann, W., "Spectroscopy of image-potential states by two-photon photoemission," Applied Physics A, vol. 49, no. 4, pp. 365-377, 1989.

Stephan Hüfner, Photoelectron Spectroscopy Principles and Applications. Springer, 2003.

Su, C., Tang, D., and Heskett, D., "Two-dimensional unoccupied electronic band structure of clean cu (110) and $(1 \times 2)$ na/cu (110)," Surface science, vol. 310, no. 1, pp. 45-51, 1994. 